\title{
2-Arylnaphthoquinone analogues: potential anti-TB and pro-apoptotic agents
}

\author{
Ivan R Green, ${ }^{\text {a* }}$ Sunil Sagar, ${ }^{\mathrm{a}}$ Wendell Swigelaar, ${ }^{\mathrm{a}}$ Farouk Ameer, ${ }^{\mathrm{a}}$ and Mervin Meyer ${ }^{\mathrm{b}}$ \\ ${ }^{a}$ Department of Chemistry, University of the Western Cape, Private Bag X17, \\ Bellville, 7530, South Africa \\ ${ }^{b}$ Department of Biotechnology, University of the Western Cape, Private Bag X17, \\ Bellville, 7530, South Africa \\ E-mail: igreen@uwc.ac.za
}

\begin{abstract}
A useful library of substituted 2-arylnaphthoquinones prepared by reaction between the corresponding bromonaphthoquinones and arylboronic acids via Suzuki-Miyaura protocols has been established. Conversion of some of the products into new analogues was effected.
\end{abstract}

Keywords: Bromonaphthoquinones, naphthylboronic acids, phenylboronic acids, SuzukiMiyaura coupling, demethylation, oxidation

\section{Introduction}

The bisnaphthoquinone disospyrin 1 comprises two 7-methyljuglone units linked between $\mathrm{C} 2$ and $\mathrm{C}^{\prime}{ }^{\prime}{ }^{1,2}$ Antimycobacterial activity studies performed on diospyrin $\mathbf{1}$ alerted the scientific community to the potential importance of this natural product ${ }^{3}$ which was soon followed by its first published synthesis by Yoshida and Mori in the same year. ${ }^{4}$ Subsequent studies, which included the synthesis and evaluation of related analogues of diospyrin $\mathbf{1}$ demonstrated the potential of this basic scaffold to be considered as an integral aspect for good antimycrobacterial activity. ${ }^{5-7}$

In order to gain a better understanding of the structural features and functional parameters that are necessary for such systems to demonstrate activity, we synthesized a number of biquinone molecules in which the two quinone moieties were directly linked viz., 2 and evaluated their pro-apoptotic activities against three human cancer cells lines. In addition the demethylated analogues viz., 3 were also evaluated and demonstrated that the molecules were specific for the available cancer cell lines evaluated..$^{8,9}$ (Figure 1). 
<smiles>Cc1cc(O)c2c(c1)C(=O)C(c1c(C)cc3c(c1O)C(=O)C=CC3=O)=CC2=O</smiles>

1<smiles>CCOc1cc(C)cc2c1C(=O)C=C(C1=CC(=O)c3c(O)cc(C)cc3C1=O)C2=O</smiles>

$2 \mathrm{R}=\mathrm{Me}$

$3 \mathrm{R}=\mathrm{H}$

Figure 1. Bisnaphthoquinones of 7-methyljuglone.

All the former studies involved use of the 7-methyljuglone moiety as one component in the quinone-quinone linked systems. It was thus considered necessary to investigate a) whether two quinone-quinone linked moieties are necessary for biological activity, b) whether the 7-methyl group was necessary to enhance any biological activity, c) what, if any, relative reactivities between the peri OMe $v s$ the peri $\mathrm{OH}$ groups are detectable and $\mathrm{d}$ ) what the nature of the substituent attached at $\mathrm{C} 2$ of the final juglone moiety should be viz., either phenyl or naphthyl. The latter investigation was prompted by numerous examples reported in the literature. ${ }^{10}$

This paper describes the syntheses of a number of families of substituted 2-phenyl- and 2naphthyl-1,4-naphthoquinones related to the diospyrin type molecules together with a very brief mention of the biological activities of a few of the more active molecules selected to emphasize the importance of this work.

\section{Results and Discussion}

In the first series of substituted 2-arylnapthoquinoidal compounds synthesized, 2-bromo-7methylnaphthoquinone $4^{8,12}$ was selected since this moiety represented the 5-deoxy analogue of the 'left hand' juglone moiety in diospyrin 1. Thus treatment of bromoquinone 4 with boronic acid 5 under Suzuki-Miyaura conditions ${ }^{13 a-c}$ afforded the expected naphthoquinone product 6 (90\%) while a similar synthetic protocol between bromoquinone $\mathbf{4}$ and boronic acid 7 afforded the naphthoquinone product $\mathbf{8}(86 \%)$. Since one of our major future goals involved the biological evaluation of analogues containing a disulfoxide nucleus to mimic a 9,10-anthraquinoidal moiety, bromoquinone $\mathbf{4}$ was coupled to the thianthrenyl boronic acid $\mathbf{9}$ to afford the desired thianthrenyl naphthoquinone $\mathbf{1 0}(82 \%)$. This was in turn was oxidized using metachloroperbenzoic acid ( $m$-CPBA) in dichloromethane under stirring to the corresponding disulfoxide $11(58 \%)$ according to the protocol of Nakayama et al. ${ }^{14}$ The IR spectrum of the

product 11 demonstrated clear $\mathrm{S}=\mathrm{O}$ stretching vibrations at 1328 and $1164 \mathrm{~cm}^{-1}$. Apart from some reasonable variations between the quite complex NMR spectra of the thianthrenyl 
naphthoquinone $\mathbf{1 0}$ and the corresponding naphthoquinone sulfoxide 11, the molecular formula of 11 viz., $\mathrm{C}_{23} \mathrm{H}_{14} \mathrm{O}_{4} \mathrm{~S}_{2}$ requires $\mathrm{M}^{+} 418.0334$ amu (found $\mathrm{M}^{+} 418.0330$ ) proved to be the major factor establishing the correct structure (Scheme 1).<smiles>Cc1ccc2c(c1)C(=O)C(Br)=CC2=O</smiles>

4<smiles>Cc1ccc2c(c1)C(=O)C(c1ccc3ccccc3c1)=CC2=O</smiles>

7

$8(86 \%)$

4<smiles>Oc1cccc2c1Sc1ccccc1S2</smiles><smiles>Cc1ccc2c(c1)C(=O)C(c1cccc3c1SC1C=CC=CC1S3)=CC2=O</smiles>

9<smiles>Cc1ccc2c(c1)C(=O)C(c1cccc3c1S(=O)C1C=CC=CC1S3=O)=CC2=O</smiles>

a) $\mathrm{Pd}\left(\mathrm{PPh}_{3}\right)_{4} /$ benzene/ $\mathrm{Na}_{2} \mathrm{CO}_{3} /$ reflux

b) $\mathrm{m}-\mathrm{CPBA} / \mathrm{CH}_{2} \mathrm{Cl}_{2}$

Scheme 1. Products from 2-bromo-7-methylnaphthoquinone and naphthyl and thianthrenyl boronic acids.

For the second series of 2-arylnaphthoquinoidal compounds related to the basic skeleton of diospyrin 1, 2-bromo-5-methoxynaphthoquinone 12 was prepared ${ }^{15,16}$ which represented the 7demethyl analogue of the 'left hand' ring. Treatment of quinone $\mathbf{1 2}$ with a range of naphthyl boronic acids under Suzuki-Miyaura conditons ${ }^{13}$ afforded the expected coupled products in yields ranging from 79-94\% (Scheme 2). Chemoselective peri demethylation was effected in the transformation of quinone 18 to $\mathbf{1 9}(60 \%)$ by treatment with $\mathrm{AlCl}_{3}$ in dichloromethane. ${ }^{8}$ 
<smiles>COc1cccc2c1C(=O)C=C(Br)C2=O</smiles><smiles>COc1cccc2c1C(=O)C=C(c1ccc(C)c3ccccc13)C2=O</smiles>
$13(81 \%)$

12<smiles></smiles>

$7 \mathrm{R}=\mathrm{H}$

$14 \mathrm{R}=\mathrm{OEt}$

$15 \mathrm{R}=\mathrm{OMe}$

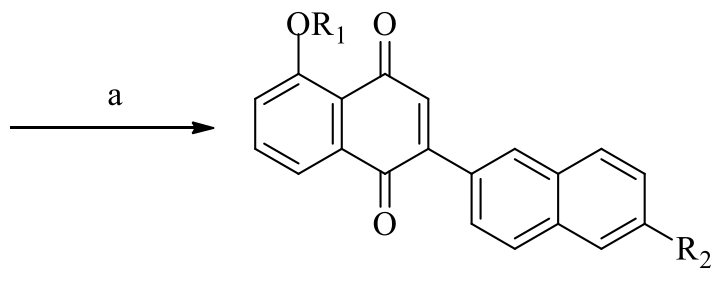

$16 \mathrm{R} 1=\mathrm{Me} ; \mathrm{R} 2=\mathrm{H}(92 \%)$

$17 \mathrm{R} 1=\mathrm{Me} ; \mathrm{R} 2=\operatorname{OEt}(88 \%)$

$18 \mathrm{R} 1=\mathrm{Me} ; \mathrm{R} 2=\mathrm{OMe}(79 \%)$

$19 \mathrm{R} 1=\mathrm{H} ; \mathrm{R} 2=\mathrm{OMe}(60 \%)$

12

$+$

9<smiles>COc1cccc2c1C(=O)C=C(c1cccc3c1Sc1ccccc1S3)C2=O</smiles>

$20(94 \%)$

a) $\mathrm{Pd}\left(\mathrm{PPh}_{3}\right)_{4} /$ benzene $/ \mathrm{Na}_{2} \mathrm{CO}_{3} /$ reflux

b) $\mathrm{m}-\mathrm{CPBA} / \mathrm{CH}_{2} \mathrm{Cl}_{2}$

c) $\mathrm{AlCl}_{3} / \mathrm{CH}_{2} \mathrm{Cl}_{2}$<smiles>COc1cccc2c1C(=O)C=C(c1cccc3c1S(=O)c1ccccc1S3=O)C2=O</smiles>

$21(68 \%)$

Scheme 2. Products from 2-bromo-5-methoxynaphthoquinone and naphthyl and thianthrenyl boronic acids.

Oxidation of the thianthrenyl naphthoquinone $\mathbf{2 0}$ to the corresponding disulfoxide $\mathbf{2 1}$ was achieved in $68 \%$ yield using the protocol described earlier. This was done to investigate the relative activities between disulfoxides $\mathbf{1 1}$ and $\mathbf{2 1}$ among others.

Literature indicates that introduction of a fluorine atom into a biologically active molecule will lead to an expectation of an increase in its biological activity profile. ${ }^{17,18}$ Treatment of 
bromoquinone 12 with the 4-fluoro- and 3,5-difluorophenylboronic acids $\mathbf{2 2}$ and 23 respectively under standard Suzuki-Miyaura conditions ${ }^{13}$ affording good yields of the corresponding naphthoquinones $\mathbf{2 5}(81 \%)$ and $\mathbf{2 6}(78 \%)$ respectively (Scheme 3).

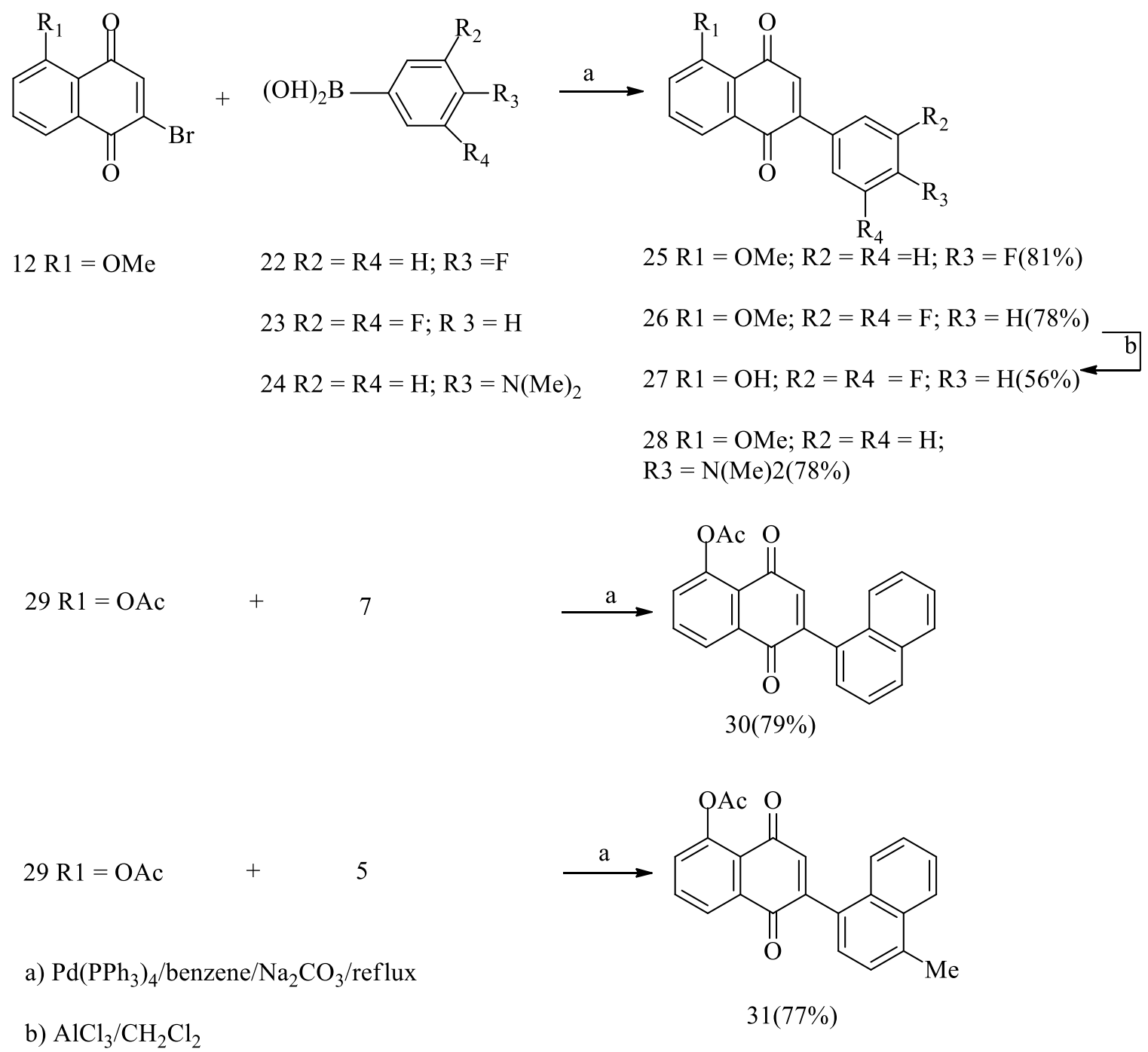

Scheme 3. Products from 2-bromo-5-methoxynaphthoquinone and fluorinated and aminated phenylboronic acids and naphthyl boronic acids.

Demethylation of the 5-methoxynaphthoquinone $\mathbf{2 6}$ to the corresponding 5-hydroxy analogue 27 was effected using $\mathrm{AlCl}_{3}$ as described earlier and would serve as a comparative model for peri hydrogen bonding influences. An example of a powerful electron-donating substituent in the 2-phenyl analogues was obtained in the form of the dimethylaminophenyl naphthoquinone $\mathbf{2 8}(\mathbf{7 8 \%})$ derived from the coupling between quinone $\mathbf{1 2}$ and boronic acid $\mathbf{2 4}$. 
<smiles>COc1cccc2c1C(=O)C(Br)=CC2=O</smiles><smiles>COc1cccc2c1C(=O)C(c1ccc3cc(C)ccc3c1)=CC2=O</smiles>

32

32

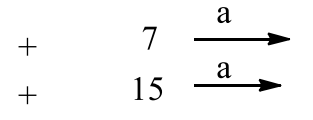

$$
\begin{aligned}
& 33 \mathrm{R}=\mathrm{H}(86 \%) \\
& 34 \mathrm{R}=\mathrm{OMe}(29 \%)
\end{aligned}
$$<smiles>COc1ccc2cc(C3=C(c4ccc5cc(OC)ccc5c4)C(=O)c4c(OC)cccc4C3=O)ccc2c1</smiles>

32

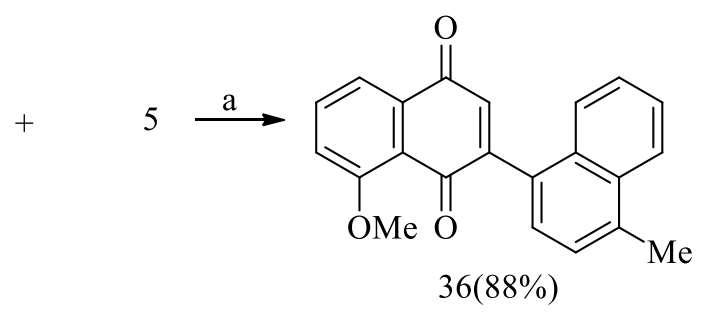

32<smiles>COc1ccc2c(c1C)C(=O)C=C(c1cccc3c1Sc1ccccc1S3)C2=O</smiles>

a) $\mathrm{Pd}\left(\mathrm{PPh}_{3}\right)_{4} /$ benzene/ $\mathrm{Na}_{2} \mathrm{CO}_{3} /$ reflux<smiles>COc1cccc2c1C(=O)C(c1cccc3c1S(=O)c1ccccc1S3=O)=CC2=O</smiles>

$38(60 \%)$

Scheme 4. Products from 2-bromo-8-methoxynaphthoquinoneand naphthyl and thianthrenyl boronic acids.

The two acetoxy analogues $\mathbf{3 0}$ and $\mathbf{3 1}$ were prepared from the corresponding acetoxybromonaphthoquinone $\mathbf{2 9}^{15}$ and boronic acids $\mathbf{7}$ and $\mathbf{5}$ respectively (Scheme 3) since we wished to evaluated what comparative electronic effects might operate in these molecules 
compared to their $\mathrm{MeO}$ isomers 16 and $\mathbf{1 3}$ respectively and how these might be manifested in their biological activities.

Studies by Lall et al. ${ }^{5}$ and Mahapatra et al. ${ }^{19}$ indicated that the peri alkoxy derivatives of diospyrin 1 and 7-methyl-5-hydroxynaphthoquinone exhibited a somewhat reduced activity against the susceptible strain of $M$. tuberculosis, viz., H37Rv. On the other hand, Chakrabarty et $a l .{ }^{20}$ reported that the peri alkoxy derivatives were more active compared to their peri hydroxyl analogues when evaluated against four human cancer cell lines.

It is for these reasons that a number of the peri alkoxy compounds were demethylated to serve as comparable test molecules for evaluations.

The isomeric peri methoxynaphthoquinone of 12, viz., $32{ }^{21}$ was then employed to synthesize the next series of naphthylnaphthoquinones and is shown in Scheme 4. Of note in this series, is the finding that reaction between naphthoquinone $\mathbf{3 2}$ and boronic acid $\mathbf{1 5}$ produced two products viz., the Suzuki product $\mathbf{3 4}$ in addition to a 2,3-disubstituted product $\mathbf{3 5}$.

In order to evaluate any variations in activity between the isomeric quinones shown in Schemes 2 and 4 due to the electronic effects of the MeO group of the quinone, the isomeric 2bromo-7-methoxynaphthoquinone $\mathbf{4 1}$ was prepared by reaction between the commercially available diene 39 and 2,5-dibromobenzoquinone 40. ${ }^{22}$ Passage of the crude adduct through a silica gel column produced a mixture of the expected product $\mathbf{4 2}(18 \%)$ together with the desired naphthoquinone 41 (50\%). Subsequently, the crude adduct obtained from the reaction described above, was methylated by treatment with methyl iodide and $\mathrm{Ag}$ (II) oxide in benzene at $24{ }^{\circ} \mathrm{C}$ in which case the desired quinone $\mathbf{4 1}$ was isolated in $80 \%$ yield (Scheme 5). Treatment of naphthoquinone $\mathbf{4 1}$ with boronic acid 15 also produced two products viz., the expected naphthoquinone 43 in addition to the 2,3-disubstitued product 44 in yields of 61 and $28 \%$ respectively similar to that found for reaction between quinone 32 and boronic acid 15 (Scheme 4). The coupled thianthrenyl naphthoquinone adduct 45, derived between reaction of naphthoquinone $\mathbf{4 1}$ and boronic acid $\mathbf{9}$, could not be oxidized to a stable product in our hands.

Bromoquinone $\mathbf{4 6}^{23}$ was selected for the final synthesis of a new series of 2arylnaphthoquinones since it represented the left hand ring of diospyrin 1. A brief series of 2naphthylnaphthoquinones having the best evaluated activity are illustrated in Scheme 6 . Demethylations and oxidation of the thianthrenyl naphthoquinone 54 into the corresponding disulfoxide 55 with $m$-CPBA are also shown. 
<smiles>C=C(C=COC)OC(C)(C)C</smiles>

39<smiles>O=C1C=C(Br)C(=O)C=C1Br</smiles>

40<smiles>[R20]c1ccc2c(c1)C(=O)C(Br)=CC2=O</smiles>

$41 \mathrm{R}=\mathrm{Me}$

41

15

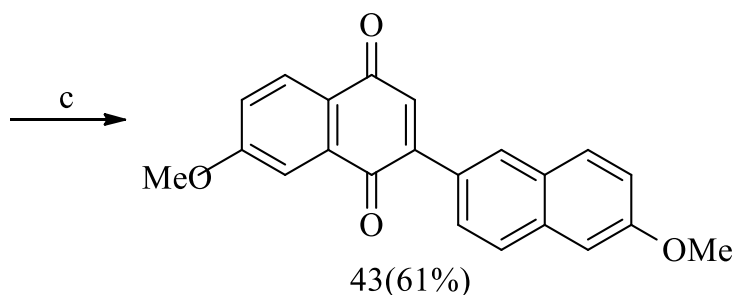

$+$<smiles>COc1ccc2c(c1)C(=O)C(c1ccc3cc(OC)ccc3c1)=C(c1ccc3cc(OC)ccc3c1)C2=O</smiles>

a) Reflux/benzene/chromatography

c) $\mathrm{Pd}\left(\mathrm{PPh}_{3}\right)_{4} /$ benzene/ $/ \mathrm{Na}_{2} \mathrm{CO}_{3} /$ reflux

b) Crude product $/ \mathrm{AgO} / \mathrm{MeI} /$ benzene
$44(28 \%)$<smiles>COc1ccc2c(c1)C(=O)C(c1cccc3c1Sc1ccccc1S3)=CC2=O</smiles>

Scheme 5. Synthesis of 2-bromo-7-methoxynaphthoquinone and its products with arylboronic acids. 
<smiles>COc1cc(C)cc2c1C(=O)C(Br)=CC2=O</smiles>

46

15<smiles>[R2]c1ccc2cc(C3=CC(=O)c4c(OCC)cc(C)cc4C3=O)ccc2c1</smiles>

$47 \mathrm{R} 1=\mathrm{Me} ; \mathrm{R} 2=\mathrm{OMe}(90 \%)$
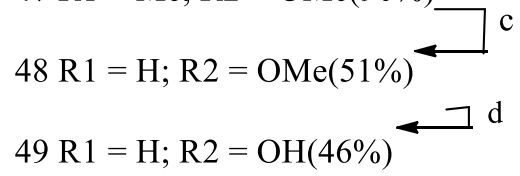

$46 \quad+\quad 7$

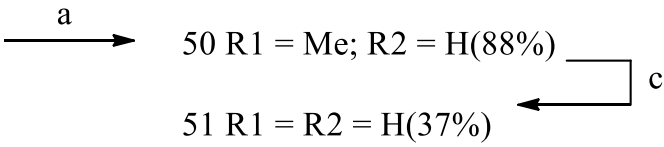

46

$+$

5
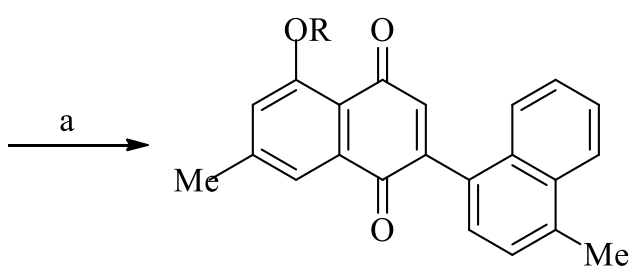

$52 \mathrm{R}=\operatorname{Me}(90 \%)$

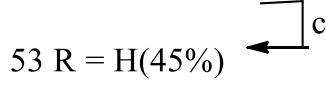

46

$+$

9

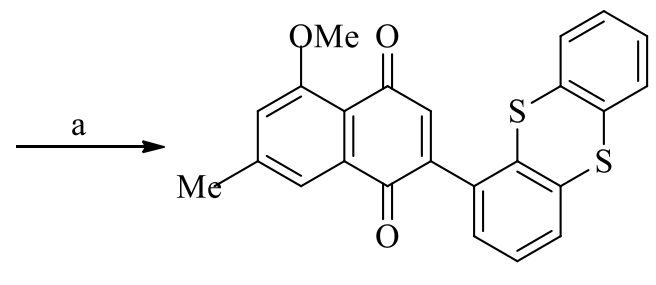

$54(86 \%)$

$\mathrm{b}$<smiles>COc1cc(C)cc2c1C(=O)C=C(c1cccc3c1S(=O)c1ccccc1S3=O)C2=O</smiles>

$55(64 \%)$

Scheme 6. Products from 2-bromo-5-methoxy-7-methylnaphthoquinone and aryl boronic acids and some transformations. 


\section{Conclusions}

A series of biologically active 2-aryl and 2-naphthylnaphthoquinones has been prepared via Suzuki Miyaura methodology and subjected to evaluations for their anti-TB and pro-apoptotic behaviour. A comprehensive article on the biological activities will be published elsewhere.

\section{Experimental Section}

General. All melting points were obtained on a Fischer Johns melting point stage and are uncorrected. ${ }^{1} \mathrm{H} \mathrm{NMR} /{ }^{13} \mathrm{C}$ NMR spectra were obtained on a $200 \mathrm{MHz} / 50 \mathrm{MHz}$ Varian Gemini 2000 spectrometer in $\mathrm{CDCl}_{3}$ with $\delta 7.26$ for ${ }^{1} \mathrm{H} \mathrm{NMR}$ and $\delta 77.11$ for ${ }^{13} \mathrm{C} \mathrm{NMR}$ spectra as internal reference. Mass spectra were performed on a Waters GCT Premier 70 eV High Resolution Mass Spectrometer and elemental analyses were performed on a Carlo Erba Strumentazione 1106 apparatus. IR spectra were recorded as nujol mulls for solids and thin films for oils on a Perkin Elmer FT-IR spectrometer Pragon 2000. Column chromatography was carried out on dry-packed columns using Merck silica gel 60 (0.063-0.2 mm) as stationary phase. The term "residue obtained upon work-up" refers to the residue obtained when the organic layer was separated, dried over $\mathrm{MgSO}_{4}$, filtered and the filtrate evaporated under rotatory evaporation. All the boronic acids were purchased from Sigma Aldrich and used without further purification. Dichloromethane (DCM) was dried by distillation from calcium chloride and stored over sodium wire while benzene was dried by distillation and stored over sodium wire.

General procedure for coupling of boronic acids with bromonaphthoquinones e.g. 2(naphthalen-2-yl)-7-methyl-1,4-naphthoquinone (8)

To a stirred (30 min) mixture of 2-bromo-7-methylnaphthoquinone $4^{8,12}$ (314 $\mathrm{mg}, 1.25 \mathrm{mmol}$ ) and $\mathrm{Pd}\left(\mathrm{PPh}_{3}\right)_{4}(148 \mathrm{mg}, 0.11 \mathrm{mmol})$ in benzene $(10 \mathrm{~mL})$ at $25^{\circ} \mathrm{C}$ under nitrogen was added an aqueous solution of sodium carbonate $(2 \mathrm{M}, 1.0 \mathrm{~mL}$ ) followed by 2-naphthaleneboronic acid 7 (203 mg, $1.18 \mathrm{mmol}$ ) in benzene $(5 \mathrm{~mL})$ drop wise. The mixture was heated under reflux for $16 \mathrm{~h}$ with vigorous stirring and after cooling to $25^{\circ} \mathrm{C}$ was quenched with water $(50 \mathrm{~mL})$ and extracted with dichloromethane. The residue obtained upon work-up was chromatographed using EtOAc: hexane (3:7) as eluent to afford naphthoquinone 8 (319 $\mathrm{mg}, 86 \%)$ as yellow needles, $\mathrm{mp}$ 169-170 ${ }^{\circ} \mathrm{C}$ (from EtOAc:hexane), IR ( $\left.v_{\max }, \mathrm{cm}^{-1}\right): 1658$ and $1652(\mathrm{C}=\mathrm{O}) .{ }^{1} \mathrm{H}$ NMR $\left(200.1 \mathrm{MHz}, \mathrm{CDCl}_{3}\right)$, $\delta_{\mathrm{H}} 2.53\left(3 \mathrm{H}, \mathrm{s}, \mathrm{CH}_{3}-7\right), 7.16(1 \mathrm{H}, \mathrm{s}, \mathrm{H}-3), 7.59$ (4H, m, H-1', H-3', H-6' and H-7'), 7.99 (6H, m, H-4', H-5', H-8', H-5, H-6 and H-8). ${ }^{13} \mathrm{C}$ NMR (50 MHz, $\left.\mathrm{CDCl}_{3}\right), \delta_{\mathrm{C}} 22.0,126.3,126.4,126.7$, $127.4,127.5,127.8,128.2,128.9$, 129.8, 130.1, 131.1, 132.6, 133.1, 134.0, 135.6, 145.1, 148.0, 185.0 and 185.1. Anal. Calcd for $\mathrm{C}_{21} \mathrm{H}_{14} \mathrm{O}_{2}$ : C, 84.54; H, 4.73. Found: C, 84.50; H, 4.82.

2-(4-Methylnaphthalen-2-yl)-7-methyl-1,4-naphthoquinone (6). Condensation between $\mathbf{4}^{8,12}$ and boronic acid 5 afforded quinone $6\left(352 \mathrm{mg}, 90 \%\right.$ ) as red needles, mp 109-111 ${ }^{\circ} \mathrm{C}$ (from EtOAc:hexane), IR ( $\left.v_{\max }, \mathrm{cm}^{-1}\right): 1660$ and $1652(\mathrm{C}=\mathrm{O}) .{ }^{1} \mathrm{H}$ NMR $\left(200.1 \mathrm{MHz}, \mathrm{CDCl}_{3}\right), \delta_{\mathrm{H}} 2.53$ 
(3H, s, $\left.\mathrm{CH}_{3}-7\right), 2.76$ (3H, s, $\left.\mathrm{CH}_{3}-4^{\prime}\right), 7.07$ (1H, s, H-3), 7.49 (6H, m, H-2', H-3', H-5', H-6', H-7' and $\left.\mathrm{H}-8^{\prime}\right), 7.97(1 \mathrm{H}, \mathrm{d}, J=0.8 \mathrm{~Hz}, \mathrm{H}-8), 8.08(1 \mathrm{H}, \mathrm{dd}, J=8.2$ and $0.8 \mathrm{~Hz}, \mathrm{H}-6), 8.10(1 \mathrm{H}, \mathrm{d}, J=$ $8.2 \mathrm{~Hz}, \mathrm{H}-5) .{ }^{13} \mathrm{C}$ NMR $\left(50 \mathrm{MHz}, \mathrm{CDCl}_{3}\right), \delta_{\mathrm{C}} 19.7,21.9,124.6,125.9,126.0,126.1,126.2$, $126.4,127.0,127.5,130.1,130.3,131.5,132.3,132.7,134.6,136.5,137.9,145.1,149.7$, 184.8,185.0. Anal. Calcd for $\mathrm{C}_{22} \mathrm{H}_{16} \mathrm{O}_{2}$ : C, 84.59; H, 5.16. Found: C, 84.50; H, 5.05.

7-Methyl-2-(thianthren-1-yl)-1,4-napthoquinone (10). Condensation between $\mathbf{4}^{8,12}$ (260 mg, $1.03 \mathrm{mmol})$ and boronic acid $9(260 \mathrm{mg}, 1.00 \mathrm{mmol})$ afforded quinone $\mathbf{1 0}(330 \mathrm{mg}, 82 \%)$ as yellow crystals, mp 208-209 ${ }^{\circ} \mathrm{C}$ (from EtOAc:hexane), IR ( $\left.v_{\max }, \mathrm{cm}^{-1}\right): 1671$ and $1660(\mathrm{C}=\mathrm{O}) .{ }^{1} \mathrm{H}$ NMR (200.1 MHz, CDCl 3 ), $\delta_{\mathrm{H}} 2.55$ (3H, s, $\left.\mathrm{CH}_{3}-7\right), 6.93$ (1H, s, H-3), 7.27 (5H, m, H-2', H-3', H-4', H-7' and H-8'), $7.51(1 \mathrm{H}, \mathrm{dd}, J=8.0$ and $1.2 \mathrm{~Hz}, \mathrm{H}-6), 7.61\left(2 \mathrm{H}, \mathrm{m}, \mathrm{H}-6^{\prime}\right.$ and $\left.\mathrm{H}-9^{\prime}\right), 8.01$ $(1 \mathrm{H}, \mathrm{d}, J=1.2 \mathrm{~Hz}, \mathrm{H}-8), 8.07(1 \mathrm{H}, \mathrm{d}, J=8.0 \mathrm{~Hz}, \mathrm{H}-5) .{ }^{13} \mathrm{C}$ NMR $\left(50 \mathrm{MHz}, \mathrm{CDCl}_{3}\right), \delta_{\mathrm{C}} 21.9$, 126.4, 126.6, 127.4, 127.6, 127.8, 127.9, 128.6 (2C), 128.9, 129.9, 130.0, 132.1, 134.1, 134.8, 135.2, 135.8, 136.2, 145.3, 148.8, 183.6, 184.8. Anal. Calcd for $\mathrm{C}_{23} \mathrm{H}_{14} \mathrm{O}_{2} \mathrm{~S}_{2}$ : C, 71.48; H, 3.65. Found: C, 71.62; H, 3.54.

2-(4-Methylnaphthalen-1-yl)-5-methoxy-1,4-naphthoquinone (13). Condensation between quinone $\mathbf{1 2}^{15,16}$ (270 $\left.\mathrm{mg}, 1.00 \mathrm{mmol}\right)$ and boronic acid 5 (190 mg. $\left.1.00 \mathrm{mmol}\right)$ afforded the quinone 13 ( $270 \mathrm{mg}, 81 \%$ ) as an orange powder, mp 203-204 ${ }^{\circ} \mathrm{C}$, IR $\left(v_{\max }, \mathrm{cm}^{-1}\right)$ : 1693 and 1667 $(\mathrm{C}=\mathrm{O}) .{ }^{1} \mathrm{H}$ NMR $\left(200.1 \mathrm{MHz}, \mathrm{CDCl}_{3}\right), \delta_{\mathrm{H}} 2.75\left(3 \mathrm{H}, \mathrm{s}, \mathrm{CH}_{3}-4^{\prime}\right), 4.07\left(3 \mathrm{H}, \mathrm{s}, \mathrm{OCH}_{3}\right), 7.02(1 \mathrm{H}, \mathrm{s}$, H-3), 7.44 (5H, m, H-6, H-2', H-3', H-6' and H-7'), 7.65 (1H, dd, $J=7.8$ and 1.4 Hz, H-5'), 7.70 $(1 \mathrm{H}, \mathrm{t}, J=8.0 \mathrm{~Hz}, \mathrm{H}-7) 7.81(1 \mathrm{H}, \mathrm{dd}, J=8.0$ and $1.6 \mathrm{~Hz}, \mathrm{H}-8), 8.06(1 \mathrm{H}, \mathrm{dd}, J=7.8$ and $1.4 \mathrm{~Hz}$, $\left.\mathrm{H}-8^{\prime}\right) .{ }^{13} \mathrm{C} \mathrm{NMR}\left(50 \mathrm{MHz}, \mathrm{CDCl}_{3}\right), \delta_{\mathrm{C}} 19.7,56.6,117.9,120.0,121.0,124.6,125.8,125.9,126.0$, 126.1, 127.0, 129.9, 131.4, 132.6, 134.7, 135.0, 136.4, 139.9,147.4, 159.6, 184.5, 184.6. Anal. Calcd for $\mathrm{C}_{22} \mathrm{H}_{17} \mathrm{O}_{3}$ : C, 80.23; H, 5.20. Found: C, 80.15; H, 5.20.

2-(Naphthalen-2-yl)-5-methoxy-1,4-naphthoquinone (16). Condensation between quinone $\mathbf{1 2}^{15,16}$ (270 mg, $\left.1.00 \mathrm{mmol}\right)$ and boronic acid $7(170 \mathrm{mg}, 1.00 \mathrm{mmol})$ afforded the quinone 16 (290 mg, 92\%) as an orange powder, mp 165-168 ${ }^{\circ} \mathrm{C}$, IR $\left(\nu_{\max }, \mathrm{cm}^{-1}\right): 1691$ and $1672(\mathrm{C}=\mathrm{O}) .{ }^{1} \mathrm{H}$ NMR (200.1 MHz, $\left.\mathrm{CDCl}_{3}\right), \delta_{\mathrm{H}} 4.05\left(3 \mathrm{H}, \mathrm{s}, \mathrm{OCH}_{3}\right), 7.13(1 \mathrm{H}, \mathrm{s}, \mathrm{H}-3), 7.35(1 \mathrm{H}, \mathrm{dd}, J=8.0$ and $1.5 \mathrm{~Hz}, \mathrm{H}-6), 7.54$ (2H, m, H-6' and H-7'), $7.65\left(1 \mathrm{H}, \mathrm{dd}, J=8.8\right.$ and $\left.1.6 \mathrm{~Hz}, \mathrm{H}-3^{\prime}\right), 7.74$ ( $1 \mathrm{H}, \mathrm{t}, J$ $=8.0 \mathrm{~Hz}, \mathrm{H}-7), 7.88\left(4 \mathrm{H}, \mathrm{m}, \mathrm{H}-4^{\prime}, \mathrm{H}-5^{\prime}, \mathrm{H}-8^{\prime}\right.$ and $\left.\mathrm{H}-8\right), 8.14\left(1 \mathrm{H}, \mathrm{d}, J=1.4 \mathrm{~Hz}, \mathrm{H}-1^{\prime}\right) .{ }^{13} \mathrm{C}$ NMR $\left(50 \mathrm{MHz}, \mathrm{CDCl}_{3}\right), \delta_{\mathrm{C}} 56.6,117.8,119.9,120.0,126.2,126.5,127.2,127.7,128.0,128.7,129.6$, 130.5, 133.0, 133.8, 134.9, 135.0, 137.5, 145.7, 154.9, 184.5, 184.8. Anal. Calcd for $\mathrm{C}_{21} \mathrm{H}_{15} \mathrm{O}_{3}$ : C, 79.98; H, 4.97. Found: C, 79.84; H, 4.82 .

2-(6-Ethoxynaphthalen-2-yl)-5-methoxy-1,4-naphthoquinone (17). Condensation between quinone $\mathbf{1 2}^{15,16}(270 \mathrm{mg}, 1.00 \mathrm{mmol})$ and boronic acid $14(220 \mathrm{mg}, 1.00 \mathrm{mmol})$ afforded the quinone $17(316 \mathrm{mg}, 88 \%)$ as a dark red powder, $\mathrm{mp}$ 144-147 ${ }^{\circ} \mathrm{C}, \mathrm{IR}\left(\mathrm{v}_{\max }, \mathrm{cm}^{-1}\right)$ : 1694 and 1679 $(\mathrm{C}=\mathrm{O}) .{ }^{1} \mathrm{H}$ NMR $\left(200.1 \mathrm{MHz}, \mathrm{CDCl}_{3}\right), \delta_{\mathrm{H}} 1.50\left(3 \mathrm{H}, \mathrm{t}, J=7.0 \mathrm{~Hz}, \mathrm{OCH}_{2} \mathrm{CH}_{3}\right), 4.05(3 \mathrm{H}, \mathrm{s}$, $\left.\mathrm{OCH}_{3}\right), 4.18\left(2 \mathrm{H}, \mathrm{q}, J=7.0 \mathrm{~Hz}, \mathrm{OCH}_{2} \mathrm{CH}_{3}\right), 7.10(1 \mathrm{H}, \mathrm{s}, \mathrm{H}-3), 7.18\left(2 \mathrm{H}, \mathrm{m}, \mathrm{H}-5^{\prime}\right.$ and H-7'), 7.33 $(1 \mathrm{H}, \mathrm{dd}, J=7.8$ and $1.5 \mathrm{~Hz}, \mathrm{H}-6), 7.61\left(1 \mathrm{H}, \mathrm{dd}, J=8.8\right.$ and $\left.1.8 \mathrm{~Hz}, \mathrm{H}-3^{\prime}\right), 7.72(1 \mathrm{H}, \mathrm{t}, J=7.8$ $\mathrm{Hz}, \mathrm{H}-7), 7.78\left(1 \mathrm{H}, \mathrm{d}, J=8.4 \mathrm{~Hz}, \mathrm{H}-8^{\prime}\right), 7.80\left(1 \mathrm{H}, \mathrm{d}, J=8.8 \mathrm{~Hz}, \mathrm{H}-4^{\prime}\right), 7.86(1 \mathrm{H}, \mathrm{dd}, J=7.8$ and $1.5 \mathrm{~Hz}, \mathrm{H}-8), 8.07\left(1 \mathrm{H}\right.$, br s, H-1'), IR $\left(v_{\max }, \mathrm{cm}^{-1}\right)$ : 1694 and $1679(\mathrm{C}=\mathrm{O}) .{ }^{13} \mathrm{C} \mathrm{NMR}(50 \mathrm{MHz}$, 
$\left.\mathrm{CDCl}_{3}\right), \delta_{\mathrm{C}} 14.8,56.5,63.8,106.4,117.7,119.7,119.9,120.1,126.6,126.9,128.1,128.5,129.5$, $130.3,134.8,135.1,135.3,136.8,145.7,158.2$, 159.4, 184.6, 185.0. Anal. Calcd for $\mathrm{C}_{23} \mathrm{H}_{19} \mathrm{O}_{4}$ : C, 76.86; H, 5.33. Found: C, 76.72; H, 5.10.

2-(6-Methoxynaphthalen-2-yl)-5-methoxy-1,4-naphthoquinone (18). Condensation between quinone $12^{15,16}$ (330 mg, $\left.1.24 \mathrm{mmol}\right)$ and boronic acid $15(250 \mathrm{mg}, 1.25 \mathrm{mmol})$ afforded the quinone 18 (340 mg, 79\%) as orange brown crystals, mp 173-176 ${ }^{\circ} \mathrm{C}, \mathrm{IR}\left(\mathrm{v}_{\max }, \mathrm{cm}^{-1}\right)$ : 1693 and $1676(\mathrm{C}=\mathrm{O}) .{ }^{1} \mathrm{H}$ NMR $\left(200.1 \mathrm{MHz}, \mathrm{CDCl}_{3}\right), \delta_{\mathrm{H}} 3.94\left(3 \mathrm{H}, \mathrm{s}, \mathrm{MeO}-66^{\prime}\right), 4.04(3 \mathrm{H}, \mathrm{s}, \mathrm{MeO}-5), 7.10$ $(1 \mathrm{H}, \mathrm{s}, \mathrm{H}-3), 7.18\left(2 \mathrm{H}, \mathrm{m}, \mathrm{H}-5^{\prime}\right.$ and H-7'), $7.34(1 \mathrm{H}, \mathrm{dd}, J=7.3$ and $1.0 \mathrm{~Hz}, \mathrm{H}-6), 7.62(1 \mathrm{H}, \mathrm{dd}, J$ $=8.4$ and $\left.1.8 \mathrm{~Hz}, \mathrm{H}-3^{\prime}\right), 7.72(1 \mathrm{H}, \mathrm{t}, J=8.4, \mathrm{H}-7), 7.80\left(2 \mathrm{H}, \mathrm{m}, \mathrm{H}-4^{\prime}\right.$ and $\left.\mathrm{H}-8^{\prime}\right), 7.86(1 \mathrm{H}$, dd, $J=$ 7.8 and $1.0 \mathrm{~Hz}, \mathrm{H}-8), 8.08\left(1 \mathrm{H}\right.$, br s, H-1'). ${ }^{13} \mathrm{C} \mathrm{NMR}\left(50 \mathrm{MHz}, \mathrm{CDCl}_{3}\right), \delta_{\mathrm{C}} 55.4,56.5,105.6$, $117.7,119.4,119.9,120.0,126.7,126.9,128.2,128.5,129.5,130.3,134.8,135.0,135.2,136.9$, 145.6, 158.8, 159.3, 184.6, 184.9. Anal. Calcd for $\mathrm{C}_{22} \mathrm{H}_{17} \mathrm{O}_{4}: \mathrm{C}, 76.51$; H, 4.96. Found: C, 76.38; H, 4.86.

5-Methoxy-2-(thianthren-1-yl)-1,4-naphthoquinone (20). Condensation between quinone $\mathbf{1 2}^{15,16}$ (270 mg, $\left.1.00 \mathrm{mmol}\right)$ and boronic acid $9(260 \mathrm{mg}, 1.00 \mathrm{mmol})$ afforded the quinone 20 (380 mg, 94\%) as a yellow powder, mp 214-217 ${ }^{\circ} \mathrm{C}$, IR $\left(v_{\max }, \mathrm{cm}^{-1}\right): 1671$ and $1660(\mathrm{C}=\mathrm{O}) .{ }^{1} \mathrm{H}$ NMR (200.1 MHz, CDCl 3 ), $\delta_{\mathrm{H}} 4.07$ (3H, s, OMe), 6.87 (1H, s, H-3), 7.25 (6H, m, H-6, H-2', H$\left.3^{\prime}, \mathrm{H}-4^{\prime}, \mathrm{H}-6^{\prime}, \mathrm{H}-7^{\prime}\right), 7.51\left(1 \mathrm{H}\right.$, dd, $J=7.6$ and $\left.1.8 \mathrm{~Hz}, \mathrm{H}-5^{\prime}\right), 7.60(1 \mathrm{H}$, dd, $J=7.6$ and $1.8 \mathrm{~Hz}$, $\left.\mathrm{H}-8^{\prime}\right), 7.75(1 \mathrm{H}, \mathrm{t}, J=8.4 \mathrm{~Hz}, \mathrm{H}-7), 7.87(1 \mathrm{H}, \mathrm{dd}, J=8.4$ and $1.8 \mathrm{~Hz}, \mathrm{H}-8) .{ }^{13} \mathrm{C}$ NMR $(50 \mathrm{MHz}$, $\left.\mathrm{CDCl}_{3}\right), \delta_{\mathrm{C}} 56.7,118.0,120.1,120.2,127.5,127.8,128.0,128.7,128.8,129.1,130.0,134.5$, 134.6, 135.2, 135.4, 135.9, 136.3, 137.1, 139.0, 146.7, 159.7, 183.7, 184.3. Anal. Calcd for $\mathrm{C}_{23} \mathrm{H}_{15} \mathrm{O}_{3} \mathrm{~S}_{2:} \mathrm{C}, 68.47 ; \mathrm{H}, 3.75$. Found: C, 68.35; H, 3.60.

2-(4-Fluorophenyl)-5-methoxy-1,4-naphthoquinone (25). Condensation between quinone $\mathbf{1 2}^{15,16}$ (270 mg, $\left.1.00 \mathrm{mmol}\right)$ and boronic acid $22(140 \mathrm{mg}, 1.00 \mathrm{mmol})$ afforded the quinone 25 (230 mg, 81\%) as orange needles, mp 184-186 ${ }^{\circ} \mathrm{C}$ (from EtOAc:hexane), IR ( $\left.v_{\max }, \mathrm{cm}^{-1}\right): 1670$ and $1663(\mathrm{C}=\mathrm{O}) .{ }^{1} \mathrm{H}$ NMR $\left(200.1 \mathrm{MHz}, \mathrm{CDCl}_{3}\right), \delta_{\mathrm{H}} 4.04(3 \mathrm{H}, \mathrm{s}, \mathrm{OMe}), 6.97(1 \mathrm{H}, \mathrm{s}, \mathrm{H}-3), 7.13$ $\left(1 \mathrm{H}, \mathrm{d}, J=8.6 \mathrm{~Hz}, \mathrm{H}-2^{\prime}\right), 7.17\left(1 \mathrm{H}, \mathrm{d}, J=8.6 \mathrm{~Hz}, \mathrm{H}-6^{\prime}\right), 7.34(1 \mathrm{H}, \mathrm{dd}, J=7.6$ and $1.2 \mathrm{~Hz}, \mathrm{H}-6)$, $7.57\left(1 \mathrm{H}, \mathrm{s}, J=8.6 \mathrm{~Hz}, \mathrm{H}-3^{\prime}\right), 7.59\left(1 \mathrm{H}, \mathrm{d}, J=8.6 \mathrm{~Hz}, \mathrm{H}-5^{\prime}\right), 7.71(1 \mathrm{H}, \mathrm{t}, J=7.6 \mathrm{~Hz}, \mathrm{H}-7), 7.83$ $(1 \mathrm{H}, \mathrm{dd}, J=7.6$ and $1.2 \mathrm{~Hz}, \mathrm{H}-8) .{ }^{13} \mathrm{C} \mathrm{NMR}\left(50 \mathrm{MHz}, \mathrm{CDCl}_{3}\right), \delta_{\mathrm{C}} 56.6,115.4,115.8,117.9$, $119.9,129.0,129.1,131.2,131.4,134.8,134.9,137.1,144.6,159.4,163.8$ (d, $J=249.1 \mathrm{~Hz}, \mathrm{C}-$ 4'), 184.3, 185.0. Anal. Calcd for $\mathrm{C}_{17} \mathrm{H}_{12} \mathrm{O}_{3} \mathrm{~F}$ : C, 72.08; H, 4.27. Found: C, 72.00; H, 4.40.

2-(3,5-Difluorophenyl)-5-methoxy-1,4-naphthoquinone (26). Condensation between quinone $\mathbf{1 2}^{15,16}$ (270 mg, $\left.1.00 \mathrm{mmol}\right)$ and boronic acid 23 (160 mg, $\left.1.00 \mathrm{mmol}\right)$ afforded the quinone 26 (230 mg, 78\%) as yellow needles, mp 221-224 ${ }^{\circ} \mathrm{C}$ (from EtOAc:hexane), IR ( $\left.v_{\max }, \mathrm{cm}^{-1}\right): 1676$ and $1667(\mathrm{C}=\mathrm{O}) .{ }^{1} \mathrm{H}$ NMR $\left(200.1 \mathrm{MHz}, \mathrm{CDCl}_{3}\right), \delta_{\mathrm{H}} 4.04(3 \mathrm{H}, \mathrm{s}, \mathrm{OMe}), 6.91(1 \mathrm{H}, \mathrm{dt}, J=8.8$ and $\left.2.2 \mathrm{~Hz}, \mathrm{H}-4^{\prime}\right), 6.99$ (1H, s, H-3), $7.13\left(2 \mathrm{H}, \mathrm{m}, \mathrm{H}-2^{\prime}\right.$ and $\left.\mathrm{H}-6^{\prime}\right), 7.35(1 \mathrm{H}$, dd, $J=8.4$ and 1,2 Hz, $\mathrm{H}-6), 7.74(1 \mathrm{H}, \mathrm{t}, J=8.4 \mathrm{~Hz}, \mathrm{H}-7), 7.84(1 \mathrm{H}, \mathrm{dd}, J=8.4$ and $1.2 \mathrm{~Hz}, \mathrm{H}-8) .{ }^{13} \mathrm{C} \mathrm{NMR}(50 \mathrm{MHz}$, $\left.\mathrm{CDCl}_{3}\right), \delta_{\mathrm{C}} 56.6,105.1\left(\mathrm{t}, J=25.1, \mathrm{C}^{\prime} 4^{\prime}\right), 112.2,112.7,118.0,119.9,120.0,134.4,135.2$ (2C), 138.1, 143.5, 159.6, $162.9\left(\mathrm{~d}, J=247.6 \mathrm{~Hz}, \mathrm{C}-3^{\prime}\right.$ and C-5'), 183.7, 183.8. Anal. Calcd for $\mathrm{C}_{17} \mathrm{H}_{11} \mathrm{O}_{3} \mathrm{~F}_{2}$ : C, 67.78; H, 3.68. Found: C, 67.90; H, 3.82. 
2-(4'-Dimethylaminophenyl)-5-methoxy-1,4-naphthoquinone (28). Condensation between quinone $\mathbf{1 2}^{15,16}(270 \mathrm{mg}, 1.00 \mathrm{mmol})$ and boronic acid $24(170 \mathrm{mg}, 1.00 \mathrm{mmol})$ afforded the quinone 28 (240 mg, 78\%) as purple needles, mp 190-192 ${ }^{\circ} \mathrm{C}$ (from EtOAc:hexane), IR ( $v_{\max }$, $\left.\mathrm{cm}^{-1}\right): 1671$ and $1660(\mathrm{C}=\mathrm{O}) .{ }^{1} \mathrm{H}$ NMR $\left(200.1 \mathrm{MHz}^{\mathrm{C}} \mathrm{CDCl}_{3}\right), \delta_{\mathrm{H}} 3.04\left(6 \mathrm{H}, \mathrm{s}, \mathrm{N}\left(\mathrm{CH}_{3}\right)_{2}\right), 4.02(3 \mathrm{H}$, s, OMe), $6.75\left(2 \mathrm{H}, \mathrm{d}, J=9.0 \mathrm{~Hz}, \mathrm{H}-3^{\prime}\right.$ and H-5'), $6.94(1 \mathrm{H}, \mathrm{s}, \mathrm{H}-3), 7.30(1 \mathrm{H}, \mathrm{dd}, J=8.4$ and 1.0 $\mathrm{Hz}, \mathrm{H}-6), 7.59$ (2H, d, $J=9.0 \mathrm{~Hz}, \mathrm{H}-2^{\prime}$ and H-6'), $7.67(1 \mathrm{H}, \mathrm{t}, J=7.6 \mathrm{~Hz}, \mathrm{H}-7), 7.82(1 \mathrm{H}, \mathrm{dd}, J=$ 8.4 and $1.0 \mathrm{~Hz}, \mathrm{H}-8) .{ }^{13} \mathrm{C} \mathrm{NMR}\left(50 \mathrm{MHz}, \mathrm{CDCl}_{3}\right), \delta_{\mathrm{C}} 40.1$ (2C), 56.5, 111.8 (2C), 117.5, 119.8, 120.2, 130.6 (2C), 133.5, 134.4, 134.5, 135.4, 145.1, 151.6, 159.2, 184.8, 185.6. Anal. Calcd for $\mathrm{C}_{19} \mathrm{H}_{18} \mathrm{NO}_{3}$ : C, 74.01; H, 5.88. Found: C, 74.22; H, 5.73.

5-Acetoxy-2-(naphthalen-2-yl)-1,4-naphthoquinone (30). Condensation between quinone $\mathbf{2 9}^{15}$ (150 mg, $0.51 \mathrm{mmol})$ and boronic acid $7(86 \mathrm{mg}, 0.50 \mathrm{mmol})$ afforded quinone 30 (137 $\mathrm{mg}$, $79 \%)$ as yellow needles, mp 182-183 ${ }^{\circ} \mathrm{C}$ (from ethanol), IR ( $\left.v_{\max }, \mathrm{cm}^{-1}\right): 1772,1668$ and 1657 $(\mathrm{C}=\mathrm{O}) .{ }^{1} \mathrm{H}$ NMR $\left(200.1 \mathrm{MHz}, \mathrm{CDCl}_{3}\right), \delta_{\mathrm{H}} 2.49\left(3 \mathrm{H}, \mathrm{s}, \mathrm{CO}_{2} \mathrm{CH}_{3}\right), 7.07(1 \mathrm{H}, \mathrm{s}, \mathrm{H}-3), 7.43$ (1H, dd, $J=8.2$ and $1.0 \mathrm{~Hz}, \mathrm{H}-6), 7.58\left(3 \mathrm{H}, \mathrm{m}, \mathrm{H}-3^{\prime}, \mathrm{H}-6^{\prime}\right.$ and $\left.\mathrm{H}-7^{\prime}\right), 7.80(1 \mathrm{H}, \mathrm{dd}, J=8.2$ and $9.2 \mathrm{~Hz}, \mathrm{H}-$ 7), $7.90\left(3 \mathrm{H}, \mathrm{m}, \mathrm{H}-4^{\prime}, \mathrm{H}-5^{\prime}\right.$ and $\left.\mathrm{H}-8^{\prime}\right), 8.11\left(1 \mathrm{H}, \mathrm{d}, J=1.0 \mathrm{~Hz}, \mathrm{H}-1^{\prime}\right), 8.18(1 \mathrm{H}, \mathrm{dd}, J=9.2$ and $1.2 \mathrm{~Hz}, \mathrm{H}-8) .{ }^{13} \mathrm{C} \mathrm{NMR}\left(50 \mathrm{MHz}, \mathrm{CDCl}_{3}\right), \delta_{\mathrm{C}} 21.1,123.5,125.6,126.1,126.6,127.4,127.7$, 128.1, 128.8, 129.5, 129.8, 130.3, 133.0, 133.9, 134.3, 134.7, 136.5, 146.9, 149.2, 169.4, 183.7, 183.8. Anal. Calcd for $\mathrm{C}_{22} \mathrm{H}_{14} \mathrm{O}_{4}$ : C, 77.18; H, 4.12. Found: C, 77.00; H, 4.20.

5-Acetoxy-2-(4-methylnaphthalen-2-yl)-1,4-naphthoquinone (31). Condensation between quinone 29 (150 mg, $0.51 \mathrm{mmol}$ ) and boronic acid 5 (93 $\mathrm{mg}, 0.50 \mathrm{mmol}$ ) affprded the quinone $31(140 \mathrm{mg}, 77 \%)$ as reddish brown needles, mp 173-174 ${ }^{\circ} \mathrm{C}$ (from ethanol), IR $\left(v_{\max }, \mathrm{cm}^{-1}\right)$ : 1769, 1667 and $1655(\mathrm{C}=\mathrm{O}) .{ }^{1} \mathrm{H}$ NMR $\left(200.1 \mathrm{MHz}, \mathrm{CDCl}_{3}\right), \delta_{\mathrm{H}} 2.49\left(3 \mathrm{H}, \mathrm{s}, \mathrm{CO}_{2} \mathrm{CH}_{3}\right), 2.75(3 \mathrm{H}$, s, $\left.\mathrm{CH}_{3}-4^{\prime}\right), 6.98(1 \mathrm{H}, \mathrm{s}, \mathrm{H}-3), 7.35\left(2 \mathrm{H}, \mathrm{m}, \mathrm{H}-2^{\prime}\right.$ and $\left.\mathrm{H}-3^{\prime}\right), 7.45(1 \mathrm{H}, \mathrm{dd}, J=8.0$ and $1.0 \mathrm{~Hz}, \mathrm{H}-$ 6), $7.54\left(2 \mathrm{H}, \mathrm{m}, \mathrm{H}-6^{\prime}\right.$ and $\left.\mathrm{H}-7^{\prime}\right), 7.65\left(1 \mathrm{H}, \mathrm{dd}, J=7.4\right.$ and $\left.1.4 \mathrm{~Hz}, \mathrm{H}-5^{\prime}\right), 7.80(1 \mathrm{H}, \mathrm{dd}, J=8.0$ and $7.6 \mathrm{~Hz}, \mathrm{H}-7), 8.07\left(1 \mathrm{H}, \mathrm{dd}, J=7.4\right.$ and $\left.1.4 \mathrm{~Hz}, \mathrm{H}-8^{\prime}\right), 8.15(1 \mathrm{H}, \mathrm{dd}, J=7.6$ and $1.0 \mathrm{~Hz}, \mathrm{H}-$ 8). ${ }^{13} \mathrm{C} \mathrm{NMR}\left(50 \mathrm{MHz}, \mathrm{CDCl}_{3}\right), \delta_{\mathrm{C}} 19.6,21.1,123.6,124.6,125.7,125.9,126.0,126.1,126.3$, 127.1, 129.6, 131.4, 132.7, 134.1, 134.8, 136.7, 138.9, 147.2, 148.6, 149.4, 169.5, 183.7(2C). Anal. Calcd for $\mathrm{C}_{23} \mathrm{H}_{16} \mathrm{O}_{4}$ : C, 77.52; H, 4.53. Found: C, 77.48; H, 4.64.

2-(Naphthalen-2-yl)-8-methoxy-1,4-naphthoquinone (33). Condensation between quinone $\mathbf{3 2}^{31}$ (270 $\mathrm{mg}, 1.00 \mathrm{mmol}$ ) and boronic acid 7 (170 mg, $1.00 \mathrm{mmol}$ ) afforded the quinone 33 (270 $\mathrm{mg}, 86 \%)$ as a yellow powder, mp 226-229 ${ }^{\circ} \mathrm{C}$, IR $\left(\nu_{\max }, \mathrm{cm}^{-1}\right): 1691$ and $1672(\mathrm{C}=\mathrm{O}) .{ }^{1} \mathrm{H}$ NMR (200.1 MHz, $\mathrm{CDCl}_{3}$ ), $\delta_{\mathrm{H}} 4.03$ (3H, s, OMe), 7.17 (1H, s, H-3), 7.35 (2H, m, H-3' and H-7), 7.58 (2H, m, H-6' and H-7'), 7.69 (4H, m, H-1', H-4', H-5' and $\left.\mathrm{H}-8^{\prime}\right), 7.75$ (1H, t, J = 7.6 Hz, H-6), $7.89(1 \mathrm{H}, \mathrm{dd}, J=7.6$ and $1.4 \mathrm{~Hz}, \mathrm{H}-5) .{ }^{13} \mathrm{C} \mathrm{NMR}\left(50 \mathrm{MHz}, \mathrm{CDCl}_{3}\right), \delta_{\mathrm{C}} 56.7,110.5,118.3,121.7$, 123.4, 124.0, 124.3, 124.5, 127.4, 129.0, 130.0, 130.8, 131.6, 133.3, 134.8, 134.9, 138.9, 143.5, 160.1, 174.4, 180.0. Anal. Calcd for $\mathrm{C}_{21} \mathrm{H}_{14} \mathrm{O}_{3}$ : C, 80.24; H, 4.49. Found: C, 80.10; H, 4.60.

2-(6-Methoxynaphthalen-2-yl)-8-methoxy-1,4-naphthoquinone (34) and 2,3-di-(6methoxynaphthalen-2-yl)-5-methoxy-1,4-naphthoquinone (35). Condensation between quinone 32 (270 mg, $1.00 \mathrm{mmol})$ and boronic acid $15(200 \mathrm{mg}, 1.00 \mathrm{mmol})$ afforded firstly quinone 34 (100 mg, 29\%) as orange crystals, mp 160-162 ${ }^{\circ} \mathrm{C}$ (from EtOAc:hexane), IR ( $v_{\max }$, 
$\left.\mathrm{cm}^{-1}\right)$ : 1663 and $1655(\mathrm{C}=\mathrm{O}) .{ }^{1} \mathrm{H}$ NMR (200.1 MHz, $\left.\mathrm{CDCl}_{3}\right), \delta_{\mathrm{H}} 3.95\left(3 \mathrm{H}, \mathrm{s}, \mathrm{MeO}-6{ }^{\prime}\right), 4.05(3 \mathrm{H}$, s, MeO-8), $7.11(1 \mathrm{H}, \mathrm{s}, \mathrm{H}-3), 7.18\left(3 \mathrm{H}, \mathrm{m}, \mathrm{H}-3^{\prime}, \mathrm{H}-5^{\prime}\right.$ and $\left.\mathrm{H}-7^{\prime}\right), 7.35(1 \mathrm{H}, \mathrm{dd}, J=8.0$ and 1.0 Hz, H-7), $7.62\left(1 \mathrm{H}, \mathrm{dd}, J=8.6\right.$ and $\left.1.8 \mathrm{~Hz}, \mathrm{H}-4^{\prime}\right), 7.71(1 \mathrm{H}, \mathrm{t}, J=8.0 \mathrm{~Hz}, \mathrm{H}-6), 7.80$ (2H, m, H$8^{\prime}$ and $\left.\mathrm{H}-5\right), 8.08\left(1 \mathrm{H}, \mathrm{d}, J=1.8 \mathrm{~Hz}, \mathrm{H}-1^{\prime}\right) .{ }^{13} \mathrm{C} \mathrm{NMR}\left(50 \mathrm{MHz}, \mathrm{CDCl}_{3}\right), \delta_{\mathrm{C}} 55.5,56.7,105.8$, $118.1,118.8,119.5,121.0,126.8,127.2$, 128.6, 129.1, 130.0, 130.5, 132.5, 134.6, 135.0, 135.4, 149.9, 159.0, 160.1, 184.3, 185.3. Anal. Calcd for $\mathrm{C}_{22} \mathrm{H}_{16} \mathrm{O}_{4}$ : C, 76.73; H, 4.68. Found: C, 76.60; $\mathrm{H}, 4.80$. Further elution gave quinone $35(210 \mathrm{mg}, 42 \%)$ as a yellow powder, $\mathrm{mp} 233-236{ }^{\circ} \mathrm{C}$, IR $\left(v_{\max }, \mathrm{cm}^{-1}\right)$ : 1668 and $1658(\mathrm{C}=\mathrm{O}) .{ }^{1} \mathrm{H}$ NMR $\left(200.1 \mathrm{MHz}, \mathrm{CDCl}_{3}\right), \delta_{\mathrm{H}} 3.86\left(6 \mathrm{H}, \mathrm{s}, \mathrm{MeO}-6^{\prime}\right.$ and MeO-6"'), 4.03 (3H, s , MeO-5), 7.05 (6H, m, H-3', H-3"', H-4', H-4"', H-5' and H-5'), 7.36 (1H, dd, $J=8.0$ and $1.4 \mathrm{~Hz}, \mathrm{H}-6), 7.45$ (2H, m, H-7' and H-7' $), 7.60$ (4H, m, H-1', H-1', H-8' and H$\left.8^{\prime \prime}\right), 7.74(1 \mathrm{H}, \mathrm{t}, J=8.0, \mathrm{H}-7), 7.88(1 \mathrm{H}, \mathrm{dd}, J=7.6$ and $1.4 \mathrm{~Hz}, \mathrm{H}-8) .{ }^{13} \mathrm{C} \mathrm{NMR}(50 \mathrm{MHz}$, $\left.\mathrm{CDCl}_{3}\right), \quad \delta_{\mathrm{C}} 55.4(2 \mathrm{C}), \quad 56.6,105.7(2 \mathrm{C}), 113.1(2 \mathrm{C}), 118.0,120.6(2 \mathrm{C}), 122.6,124.4(2 \mathrm{C})$, 124.6(2C), 126.1, 127.1(2C), 130.5(2C), 132.9, 134.9, 135.0, 137.0, 137.1, 137.9, 138.0(2C), 154.8(2C), 161.3, 184.4, 185.1. Anal. Calcd for $\mathrm{C}_{33} \mathrm{H}_{24} \mathrm{O}_{5}$ : C, 79.19; H, 4.83. Found: C, 79.00; H, 4.72 .

2-(4-Methylnaphthalen-1-yl)-8-methoxy-1,4-naphthoquinone (36). Condensation between quinone 32 (270 mg, $1.00 \mathrm{mmol})$ and boronic acid $5(190 \mathrm{mg}, 1.00 \mathrm{mmol})$ afforded the quinone $36(290 \mathrm{mg}, 88 \%)$ as an orange powder, $\mathrm{mp} 157-160{ }^{\circ} \mathrm{C}, \mathrm{IR}\left(\nu_{\max }, \mathrm{cm}^{-1}\right): 1695$ and $1673(\mathrm{C}=\mathrm{O})$. ${ }^{1} \mathrm{H}$ NMR (200.1 MHz, CDCl 3$), \delta_{\mathrm{H}} 2.75$ (3H, s, CH $\left.3-4{ }^{\prime}\right), 3.97$ (3H, s, OMe), $7.02(1 \mathrm{H}, \mathrm{s}, \mathrm{H}-3)$, $7.40\left(5 \mathrm{H}, \mathrm{m}, \mathrm{H}-6, \mathrm{H}-2^{\prime}, \mathrm{H}-3^{\prime}, \mathrm{H}-6^{\prime}\right.$ and H-7'), $7.68\left(1 \mathrm{H}, \mathrm{dd}, J=7.8\right.$ and $\left.1.4 \mathrm{~Hz}, \mathrm{H}-5^{\prime}\right), 7.74(1 \mathrm{H}$, $\mathrm{t}, J=7.6 \mathrm{~Hz}, \mathrm{H}-6), 7.84(1 \mathrm{H}, \mathrm{dd}, J=7.6$ and $1.4 \mathrm{~Hz}, \mathrm{H}-5), 8.05(1 \mathrm{H}, \mathrm{dd}, J=7.8$ and $1.4 \mathrm{~Hz}, \mathrm{H}-$ $\left.8^{\prime}\right) .{ }^{13} \mathrm{C} \mathrm{NMR}\left(50 \mathrm{MHz}, \mathrm{CDCl}_{3}\right), \delta_{\mathrm{C}} 19.6,56.5,118.1,118.9,120.4,124.5,125.8,125.9,126.1$, 126.2, 127.0, 130.7, 131.6, 132.6, 134.5, 134.9, 135.6, 136.2,151.6, 160.1, 183.6, 185.3. Anal. Calcd for $\mathrm{C}_{22} \mathrm{H}_{16} \mathrm{O}_{3}:$ C, 80.47; H, 4.91. Found: C, 80.33; H, 4.98 .

8-Methoxy-2-(thianthren-1-yl)-1,4-naphthoquinone (37). Condensation between quinone 32 (270 $\mathrm{mg}, 1.00 \mathrm{mmol}$ ) and boronic acid $9(260 \mathrm{mg}, 1.00 \mathrm{mmol})$ afforded the quinone 37 (350 $\mathrm{mg}$, $86 \%)$ as a yellow powder, mp 195-198 ${ }^{\circ} \mathrm{C}$, IR $\left(v_{\max }, \mathrm{cm}^{-1}\right): 1688$ and $1670(\mathrm{C}=\mathrm{O}) .{ }^{1} \mathrm{H}$ NMR (200.1 MHz, CDCl 3 ), $\delta_{\mathrm{H}} 4.02$ (3H, s, OMe), 6.88 (1H, s, H-3), 7.30 (6H, m, H-7, H-2', H-3', H4', H-6' and $\left.\mathrm{H}-7^{\prime}\right), 7.50\left(1 \mathrm{H}, \mathrm{dd}, J=7.6\right.$ and $\left.1.8 \mathrm{~Hz}, \mathrm{H}-5^{\prime}\right), 7.59\left(1 \mathrm{H}, \mathrm{dd}, 7.6\right.$ and $\left.1.8 \mathrm{~Hz}, \mathrm{H}-8^{\prime}\right)$, $7.74(1 \mathrm{H}, \mathrm{t}, J=8.0 \mathrm{~Hz}, \mathrm{H}-6), 7.82(1 \mathrm{H}, \mathrm{dd}, J=8.0$ and $1,6 \mathrm{~Hz}, \mathrm{H}-5) .{ }^{13} \mathrm{C} \mathrm{NMR}(50 \mathrm{MHz}$, $\left.\mathrm{CDCl}_{3}\right), \delta_{\mathrm{C}} 56.5,118.3,118.9,120.5,127.2,127.7,127.8,127.9,128.6,128.7,129.0,129.7$, $134.4,134.5,134.9,135.2,135.7,136.3,136.8,150.7,160.1,182.6,185.1$. Anal. Calcd for $\mathrm{C}_{23} \mathrm{H}_{14} \mathrm{O}_{3} \mathrm{~S}_{2}$ : C, 68.64; H, 3.51. Found: C, 68.58; H, 3.44.

\section{2-Bromo-7-methoxy-1,4-naphthoquinone (41) and 2-bromo-7-hydroxy-1,4-naphthoquinone} (42). To a stirred solution of 2,5-dibromobenzoquinone 40 (6.38 g, $24 \mathrm{mmol})$ in dry benzene (60 $\mathrm{mL})$ at $25^{\circ} \mathrm{C}$ was dripped in 1-methoxy-3-trimethylsilyloxybutadiene 39 (5 g, $29.2 \mathrm{mmol}$ ) over a period of $30 \mathrm{~min}$ and stirring was continued at $50{ }^{\circ} \mathrm{C}$ until starting material had been consumed (tlc). The residue obtained upon removal of all solvents was chromatographed and eluted with EtOAc:hexane (3:7) as eluent to yield firstly quinone $41(1.18 \mathrm{~g}, 18 \%)$ as yellow crystals, $\mathrm{mp}$ 134- $135^{\circ} \mathrm{C}$ (from ethanol), IR ( $\left.v_{\max }, \mathrm{cm}^{-1}\right): 1670(\mathrm{C}=\mathrm{O}) .{ }^{1} \mathrm{H}$ NMR $\left(200.1 \mathrm{MHz}, \mathrm{CDCl}_{3}\right), \delta_{\mathrm{H}} 3.96$ 
$(3 \mathrm{H}, \mathrm{s}, \mathrm{OMe}), 7.23(1 \mathrm{H}, \mathrm{dd}, J=8.6$ and $2.6 \mathrm{~Hz}, \mathrm{H}-6), 7.26(2 \mathrm{H}, \mathrm{s}, \mathrm{H}-3), 7.60(1 \mathrm{H}, \mathrm{d}, J=2.6 \mathrm{~Hz}$, $\mathrm{H}-8), 8.12(1 \mathrm{H}, \mathrm{d}, J=8.6 \mathrm{~Hz}, \mathrm{H}-5) .{ }^{13} \mathrm{C} \mathrm{NMR}\left(50 \mathrm{MHz}, \mathrm{CDCl}_{3}\right), \delta_{\mathrm{C}} 56.2,111.9,121.0,129.4$, 130.8, 133.0, 140.7, 141.7, 164.7, 174.9, 176.1. Anal. Calcd for $\mathrm{C}_{11} \mathrm{H}_{7} \mathrm{BrO}_{3}$ : C, 49.47; H, 2.64 . Found: C, 49.64; H, 2.53. Further elution of the column yielded the quinone $\mathbf{4 2}(3.06 \mathrm{~g}, 50 \%)$ as dark orange crystals, mp 233-235 ${ }^{\circ} \mathrm{C}$ (from EtOAc:hexane), IR $\left(v_{\max }, \mathrm{cm}^{-1}\right): 3320(\mathrm{OH})$ and 1690 $(\mathrm{C}=\mathrm{O}) .{ }^{1} \mathrm{H}$ NMR $\left(200.1 \mathrm{MHz}\right.$, Acetone-d $\left.\mathrm{d}_{6}\right), \delta_{\mathrm{H}} 7.16(1 \mathrm{H}, \mathrm{s}, \mathrm{H}-3), 7.28(1 \mathrm{H}, \mathrm{dd}, J=8.8$ and 2.4 $\mathrm{Hz}, \mathrm{H}-6), 7.51(1 \mathrm{H}, \mathrm{d}, J=2.4 \mathrm{~Hz}, \mathrm{H}-8), 8.03(1 \mathrm{H}, \mathrm{t}, J=8.8 \mathrm{~Hz}, \mathrm{H}-5), 10.00(1 \mathrm{H}, \mathrm{s}, \mathrm{OH}) .{ }^{13} \mathrm{C}$ NMR (50 MHz, $\left.\mathrm{CDCl}_{3}\right), \delta_{\mathrm{C}} 114.6,122.2,124.3,131.6,134.2,142.1,143.7,163.9,175.4,176.7$. Anal. Calcd for $\mathrm{C}_{10} \mathrm{H}_{5} \mathrm{BrO}_{3}$ : C, 47.46; H, 1.99. Found: C, 47.76; H, 2.20.

In a repeat experiment based on the same quantities, the crude product was dissolved in benzene $(100 \mathrm{~mL})$ containing $\mathrm{AgO}(10 \mathrm{~g})$ and methyl iodide $(13.63 \mathrm{~g}, 96 \mathrm{mmol})$ and stirred at $25{ }^{\circ} \mathrm{C}$ for $24 \mathrm{~h}$, filtered and the residue chromatographed using EtOAc:hexane (3:7) as eluent to yield the quinone product $41(5.13 \mathrm{~g}, 80 \%)$.

2-(6-Methoxynaphthalen-2-yl)-7-methoxy-1,4-naphthoquinone (43) and 2,3-di(6methoxynaphthalen-2-yl)-6-methoxy-1,4-naphthoquinone (44). Condensation between quinone 41 (270 mg, $1.00 \mathrm{mmol}$ ) and boronic acid 15 (200 mg, $1.00 \mathrm{mmol})$ afforded firstly the quinone $43(210 \mathrm{mg}, 61 \%)$ as a yellow powder, $\mathrm{mp} 159-162{ }^{\circ} \mathrm{C}, \mathrm{IR}\left(\mathrm{v}_{\max }, \mathrm{cm}^{-1}\right): 1663$ and 1655 $(\mathrm{C}=\mathrm{O}) .{ }^{1} \mathrm{H}$ NMR $\left(200.1 \mathrm{MHz}, \mathrm{CDCl}_{3}\right), \delta_{\mathrm{H}} 3.95$ (3H, s, MeO-6'), 3.98 (3H, s, MeO-7), $7.12(1 \mathrm{H}$, s, H-3), $7.20\left(2 \mathrm{H}, \mathrm{m}, \mathrm{H}-5^{\prime}\right.$ and H-7'), $7.27(1 \mathrm{H}, \mathrm{dd}, J=8.4$ and $2.6 \mathrm{~Hz}, \mathrm{H}-6), 7.62(1 \mathrm{H}, \mathrm{dd}, J=$ 8.4 and $\left.1.4 \mathrm{~Hz}, \mathrm{H}-3^{\prime}\right), 7.64(1 \mathrm{H}, \mathrm{d}, J=2.6 \mathrm{~Hz}, \mathrm{H}-8), 7.81\left(2 \mathrm{H}, \mathrm{d}, J=8.4 \mathrm{~Hz}, \mathrm{H}-4^{\prime}\right.$ and $\left.\mathrm{H}-8^{\prime}\right), 8.06$ $\left(1 \mathrm{H}, \mathrm{d}, J=1.4 \mathrm{~Hz}, \mathrm{H}-1^{\prime}\right), 8.08(1 \mathrm{H}, \mathrm{d}, J=8.4 \mathrm{~Hz}, \mathrm{H}-5) .{ }^{13} \mathrm{C} \mathrm{NMR}\left(50 \mathrm{MHz}, \mathrm{CDCl}_{3}\right), \delta_{\mathrm{C}} 55.4$, $55.9,105.6,110.5,119.5,120.3,125.9,126.8,126.9,128.4,128.5,128.6,129.6,130.4,135.1$, 135.3, 147.5, 147.7, 158.9, 164.2, 184.3, 184.9. Anal. Calcd for $\mathrm{C}_{22} \mathrm{H}_{16} \mathrm{O}_{4}: \mathrm{C}, 76.73 ; \mathrm{H}, 4.68$. Found: C, 76.58; H, 4.56. Further elution afforded quinone 44 (140 mg, 28\%) as bright orange crystals, mp 179-181 ${ }^{\circ} \mathrm{C}$, IR ( $\left.v_{\max }, \mathrm{cm}^{-1}\right): 1668$ and $1658(\mathrm{C}=\mathrm{O}) .{ }^{1} \mathrm{H}$ NMR $\left(200.1 \mathrm{MHz}, \mathrm{CDCl}_{3}\right)$, $\delta_{\mathrm{H}} 3.87$ (6H, s, MeO-6' and MeO-6"'), 3.98 (3H, s, MeO-6), 7.10 (6H, m, H-3', H-3"', H-4', H-4"', H-5', H-5'), $7.28(1 \mathrm{H}, \mathrm{dd}, J=8.8$ and $2.6 \mathrm{~Hz}, \mathrm{H}-7), 7.47\left(2 \mathrm{H}, \mathrm{d}, J=8.2 \mathrm{~Hz}, \mathrm{H}-7^{\prime}\right.$ and $\left.\mathrm{H}-7^{\prime \prime}\right)$, 7.59 (4H, m, H-1', H-1"', H-8', H-8'), $7.65(1 \mathrm{H}, \mathrm{d}, J=2.6 \mathrm{~Hz}, \mathrm{H}-5), 8.18(1 \mathrm{H}, \mathrm{d}, J=8.8 \mathrm{~Hz}, \mathrm{H}-$ 8). ${ }^{13} \mathrm{C} \mathrm{NMR}\left(50 \mathrm{MHz}, \mathrm{CDCl}_{3}\right), \delta_{\mathrm{C}} 55.4(2 \mathrm{C}), 56.1,105.7(2 \mathrm{C}), 109.8,118.9(2 \mathrm{C}), 120.7,126.0$, $126.1,128.2$, 128.3, 128.7(4C), 129.3, 130.0, 130.1, 130.8, 130.9, 134.2(2C), 134.4(2C), 145.3, 145.7, 158.4(2C), 164.3, 184.2, 185.2. Anal. Calcd for $\mathrm{C}_{33} \mathrm{H}_{24} \mathrm{O}_{5}$ : C, 79.19; H, 4.83. Found: C, 79.10; H, 5.00.

7-Methoxy-2-(thianthren-1-yl)-1,4-naphthoquinone (45). Condensation between quinone 41 (270 mg, $1.00 \mathrm{mmol}$ ) and boronic acid 9 (260 mg, $1.00 \mathrm{mmol})$ afforded the quinone 45 (320 $\mathrm{mg}$, $79 \%)$ as a yellow powder, mp 184-187 ${ }^{\circ} \mathrm{C}$, IR $\left(v_{\max }, \mathrm{cm}^{-1}\right): 1688$ and $1670(\mathrm{C}=\mathrm{O}) .{ }^{1} \mathrm{H}$ NMR $\left(200.1 \mathrm{MHz}, \mathrm{CDCl}_{3}\right), \delta_{\mathrm{H}} 3.99$ (1H, s, OMe), 6.90 (1H, s, H-3), 7.25 (6H, m, H-6, H-2', H-3', H4', H-6', H-7'), $7.50\left(1 \mathrm{H}\right.$, dd, $J=7.6$ and $\left.1.4 \mathrm{~Hz}, \mathrm{H}-5^{\prime}\right), 7.61\left(1 \mathrm{H}, \mathrm{dd}, J=7.6\right.$ and $\left.1.4 \mathrm{~Hz}, \mathrm{H}-8^{\prime}\right)$, $7.64(1 \mathrm{H}, \mathrm{d}, J=2.6 \mathrm{~Hz}, \mathrm{H}-8), 8.12(1 \mathrm{H}, \mathrm{d}, J=8.4 \mathrm{~Hz}, \mathrm{H}-5) .{ }^{13} \mathrm{C}$ NMR $\left(50 \mathrm{MHz}, \mathrm{CDCl}_{3}\right), \delta_{\mathrm{C}}$ $56.0,110.6,120.5,127.4,127.8,128.0,128.6,128.8,129.0,129.9,130.3,134.3,134.7,134.8$, 
135.0, 135.1, 135.2, 136.2, 137.2, 148.6, 164.4, 183.5,184.0. Anal. Calcd for $\mathrm{C}_{23} \mathrm{H}_{14} \mathrm{O}_{3} \mathrm{~S}_{2}$ : C, 68.64; H, 3.51. Found: C, 68.50; H, 3.50.

2-(6-Methoxynaphthalen-2-yl)-5-methoxy-7-methyl-1,4-naphthoquinone (47). Condensation between quinone 46 (351 mg, $1.25 \mathrm{mmol}$ ) and boronic acid 15 (238 mg, $1.18 \mathrm{mmol}$ ) afforded the quinone 47 (401 mg, 90\%) as orange-red needles, mp 179-181 ${ }^{\circ} \mathrm{C}$ (from ethanol), IR $\left(v_{\max }, \mathrm{cm}^{-}\right.$ $\left.{ }^{1}\right): 1663$ and $1645(\mathrm{C}=\mathrm{O}) .{ }^{1} \mathrm{H}$ NMR $\left(200.1 \mathrm{MHz} \mathrm{CDCl}_{3}\right), \delta_{\mathrm{H}} 2.51\left(3 \mathrm{H}, \mathrm{s}, \mathrm{CH}_{3}-7\right), 3.95(3 \mathrm{H}, \mathrm{s}$, MeO-6'), 4.03 (3H, s, MeO-5), 7.07 (1H, s, H-3), 7.13 (1H, d, J=1.2 Hz, H-6), 7.18 (2H, m, H-

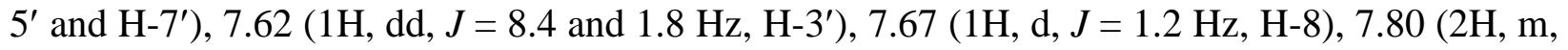
$\mathrm{H}-4^{\prime}$ and $\left.\mathrm{H}-8^{\prime}\right), 8.07\left(1 \mathrm{H}, \mathrm{d}, J=1.8 \mathrm{~Hz}, \mathrm{H}-1^{\prime}\right) .{ }^{13} \mathrm{C} \mathrm{NMR}\left(50 \mathrm{MHz}, \mathrm{CDCl}_{3}\right), \delta_{\mathrm{C}} 22.5,55.5,56.6$, $105.8,118.3,119.5,120.8,121.9,126.9$, 127.0, 128.4, 128.6, 129.5, 130.4, 135.0, 135.3, 137.2, 145.6, 146,3, 158.9, 159.7, 184.4, 185.3. Anal. Calcd for $\mathrm{C}_{23} \mathrm{H}_{18} \mathrm{O}_{4}$ : C, 77.08, H, 5.06. Found: C, $77.20, \mathrm{H}, 5.22$.

2-(Naphthalen-2-yl)-5-methoxy-7-methyl-1,4-naphthoquinone (50). Condensation between quinone 46 (351 mg, $1.25 \mathrm{mmol})$ and boronic acid 7 (203 $\mathrm{mg}, 1.18 \mathrm{mmol})$ afforded the quinone 50 (358 $\mathrm{mg}, 88 \%)$ as a yellow powder, $\mathrm{mp} 172-173{ }^{\circ} \mathrm{C}, \mathrm{IR}\left(v_{\max }, \mathrm{cm}^{-1}\right)$ : 1658 and $1651(\mathrm{C}=\mathrm{O})$. ${ }^{1} \mathrm{H}$ NMR (200.1 MHz, CDCl 3 ), $\delta_{\mathrm{H}} 2.54\left(3 \mathrm{H}, \mathrm{s}, \mathrm{CH}_{3}-7\right), 4.03(3 \mathrm{H}, \mathrm{s}, \mathrm{MeO}-5), 7.09$ (1H, s, H-3), $7.13(1 \mathrm{H}, \mathrm{d}, J=1.4 \mathrm{~Hz}, \mathrm{H}-6), 7.54$ (2H, m, H-6' and H-7'), 7.66 (2H, m, H-5' and H-8'), 7.89 $\left(3 \mathrm{H}, \mathrm{m}, \mathrm{H}-1^{\prime}, \mathrm{H}-3^{\prime}\right.$ and H-4'), $8.13(1 \mathrm{H}, \mathrm{d}, J=1.4 \mathrm{~Hz}, \mathrm{H}-8) .{ }^{13} \mathrm{C} \mathrm{NMR}\left(50 \mathrm{MHz}, \mathrm{CDCl}_{3}\right), \delta_{\mathrm{C}} 22.5$, 56.6, 118.3, 120.8, 126.3, 126.6, 127.3, 127.8, 128.1, 128.8, 129.6, 130.8, 133.2, 133.9, 134.8, 137.8, 145.7, 146.5, 159.7, 184.3, 185.2. Anal. Calcd for $\mathrm{C}_{22} \mathrm{H}_{16} \mathrm{O}_{3}$ : C, 80.47; H, 4.91. Found: C, 80.70; H, 4.83 .

5-Methoxy-7-methyl-2-(4-methylnaphthalen-2-yl)-1,4-naphthoquinone (52). Condensation between quinone 46 (351 $\mathrm{mg}, 1.25 \mathrm{mmol})$ and boronic acid 5 (220 mg, $1.18 \mathrm{mmol})$ afforded the quinone 52 (428 mg, 90\%) as yellow needles (from EtOAc:hexane), mp 229-230 ${ }^{\circ} \mathrm{C}$, IR ( $v_{\max }$, $\left.\mathrm{cm}^{-1}\right)$ : 1659 and $1648(\mathrm{C}=\mathrm{O}) .{ }^{1} \mathrm{H}$ NMR $\left(200.1 \mathrm{MHz}, \mathrm{CDCl}_{3}\right), \delta_{\mathrm{H}} 2.51\left(3 \mathrm{H}, \mathrm{s}, \mathrm{CH}_{3}-7\right), 2.74(3 \mathrm{H}, \mathrm{s}$, $\left.\mathrm{CH}_{3}-4^{\prime}\right), 4.04$ (3H, s, MeO-5), 6.98 (1H, s, H-3), 7.15 (1H, d, J=1.0 Hz, H-6), 7.44 ( 4H, m, H$2^{\prime}, \mathrm{H}-3^{\prime}, \mathrm{H}-6{ }^{\prime}$ and $\left.\mathrm{H}-7^{\prime}\right), 7.65\left(2 \mathrm{H}, \mathrm{m}, \mathrm{H}-5^{\prime}\right.$ and $\left.\mathrm{H}-8\right), 8.06\left(1 \mathrm{H}, \mathrm{dd}, J=8.0\right.$ and $\left.1.0 \mathrm{~Hz}, \mathrm{H}-8^{\prime}\right) .{ }^{13} \mathrm{C}$ NMR $\left(50 \mathrm{MHz}, \mathrm{CDCl}_{3}\right), \delta_{\mathrm{C}} 19.7,22.4,56.6,118.1,118.4,120.9,124.7,126.01,126.04,126.1$, 126.2, 127.2, 130.2, 131.6, 132.7, 134.5, 136.4, 140.1, 146.5, 147.4, 159.8, 184.3, 185.0. Anal. Calcd for $\mathrm{C}_{23} \mathrm{H}_{18} \mathrm{O}_{3}$ : C, 80.68; H, 5.30. Found: C, 80.83; H, 5.45.

5-Methoxy-7-methyl-2-(thianthren-1-yl)-1,4-naphthoquinone (54). Condensation between quinone 46 (351 mg, $1.25 \mathrm{mmol})$ and boronic acid 9 (306 mg, $1.18 \mathrm{mmol})$ afforded the quinone 54 (420 mg, 86\%) as yellow needles, mp 203-204 ${ }^{\circ} \mathrm{C}$ (from EtOAc:Hexane), IR $\left(v_{\max }, \mathrm{cm}^{-1}\right)$ : 1670 and $1658(\mathrm{C}=\mathrm{O}) .{ }^{1} \mathrm{H} \mathrm{NMR}\left(200.1 \mathrm{MHz}, \mathrm{CDCl}_{3}\right), \delta_{\mathrm{H}} 2.52\left(3 \mathrm{H}, \mathrm{s}, \mathrm{CH}_{3}-7\right), 4.04(3 \mathrm{H}, \mathrm{s}$, OMe), 6.83 (1H, d, J=1.4 Hz, H-6), 7.16 ( 1H, s, H-3), 7.27 ( 5H, m, H-2', H-3', H-4', H-7' and $\left.\mathrm{H}-8^{\prime}\right), 7.49\left(1 \mathrm{H}, \mathrm{dd}, J=7.4\right.$ and $\left.1.4 \mathrm{~Hz}, \mathrm{H}-6^{\prime}\right), 7.59\left(1 \mathrm{H}, \mathrm{dd}, J=7.6\right.$ and $\left.1.6 \mathrm{~Hz}, \mathrm{H}-9^{\prime}\right), 7.67(1 \mathrm{H}$, $\mathrm{d}, J=1.4 \mathrm{~Hz}, \mathrm{H}-8) .{ }^{13} \mathrm{C} \mathrm{NMR}\left(50 \mathrm{MHz}, \mathrm{CDCl}_{3}\right), \delta 22.3,56.5,118.4,120.8,127.4,127.7,127.9$, 128.3, 128.6, 128.7, 128.9, 129.8, 131.5, 134.2, 134.6, 135.3, 136.2, 136.9, 139.0, 146.4, 146.6, 159.8, 183.8, 183.9. Anal. Calcd for $\mathrm{C}_{24} \mathrm{H}_{16} \mathrm{O}_{3} \mathrm{~S}_{2}$ : C, 69.21; H, 3.87. Found: C, 69.44; H, 3.78. 
General procedure for demethylation of the peri-methoxy group in the naphthoquinone products using $\mathrm{AlCl}_{3}$

To a solution of the naphthoquinone $(0.50 \mathrm{mmol})$ in dry DCM $(20 \mathrm{~mL})$ at $25^{\circ} \mathrm{C}$ was added $\mathrm{AlCl}_{3}$ $(2.61 \mathrm{~g}, 19.6 \mathrm{mmol})$ and the mixture stirred at $25^{\circ} \mathrm{C}$ for $24 \mathrm{~h}$ and poured into ice/water $(100 \mathrm{~mL})$. To this was added $0.1 \mathrm{M} \mathrm{HCl}(100 \mathrm{~mL})$ after which the solution was extracted with DCM (3x60 $\mathrm{mL})$. The residue obtained upon workup was chromatographed on silica gel using EtOAc:hexane (3:7) as eluent to provide the naphthol product.

2-(6-Methoxynaphthalen-2-yl)-5-hydroxy-1,4-naphthoquinone (19). Demethylation of quinone 18 (170 mg, $0.50 \mathrm{mmol})$ afforded naphthol $19(100 \mathrm{mg}, 60 \%)$ as orange-brown crystals, mp 211-214 ${ }^{\circ} \mathrm{C}$ (from EtOAc:hexane), IR $\left(v_{\max }, \mathrm{cm}^{-1}\right)$ : 3300-2700 $(\mathrm{OH}), 1687$ and $1668(\mathrm{C}=\mathrm{O})$. ${ }^{1} \mathrm{H}$ NMR (200.1 MHz, CDCl 3 ), $\delta_{\mathrm{H}} 3.96$ (3H, s, MeO-6'), 7.14 (1H, s, H-3), 7.20 (2H, m, H-5' and $\left.\mathrm{H}^{-} 7^{\prime}\right), 7.30(1 \mathrm{H}, \mathrm{dd}, J=8.0$ and $1.0 \mathrm{~Hz}, \mathrm{H}-6), 7.62\left(1 \mathrm{H}, \mathrm{dd}, J=8.4\right.$ and $\left.1.8 \mathrm{~Hz}, \mathrm{H}-3^{\prime}\right), 7.66$ $(1 \mathrm{H}, \mathrm{t}, J=8.0, \mathrm{H}-7), 7.74(1 \mathrm{H}, \mathrm{dd}, J=8.0$ and $1.0 \mathrm{~Hz}, \mathrm{H}-8), 7.81\left(2 \mathrm{H}, \mathrm{d}, J=8.4 \mathrm{~Hz}, \mathrm{H}-4^{\prime}\right.$ and H$\left.8^{\prime}\right), 8.09$ (1H, bs, H-1'), 12.09 (1H, s, HO-5). ${ }^{13} \mathrm{C} \mathrm{NMR}\left(50 \mathrm{MHz}, \mathrm{CDCl}_{3}\right), \delta_{\mathrm{C}} 55.5,105.6,115.4$, 119.7, 119.9, 124.3, 126.8, 127.1, 128.3, 128.6, 130.1, 130.6, 132.7, 134.5, 135.7, 136.4, 149.2, 159.2, 161.2, 184.2, 190.3. Anal. Calcd for $\mathrm{C}_{21} \mathrm{H}_{14} \mathrm{O}_{4}$ : C, 76.36; H, 4.27. Found: C, 76.22; H, 4.43.

2-(3,5-Difluorobenzen-1-yl)-5-hydroxy-1,4-naphthoquinone (27). Demethylation of quinone 26 (150 mg, $0.50 \mathrm{mmol})$ afforded naphthol $27(80 \mathrm{mg}, 56 \%)$ as yellow needles, $\mathrm{mp} \mathrm{220-220}{ }^{\circ} \mathrm{C}$ (from EtOAc:hexane), IR $\left(v_{\max }, \mathrm{cm}^{-1}\right): 3430(\mathrm{OH}), 1687$ and $1668(\mathrm{C}=\mathrm{O}) .{ }^{1} \mathrm{H}$ NMR $(200.1 \mathrm{MHz}$, $\left.\mathrm{CDCl}_{3}\right), \delta_{\mathrm{H}} 6.95\left(1 \mathrm{H}, \mathrm{dt}, J=8.8\right.$ and $\left.2.6 \mathrm{~Hz}, \mathrm{H}-4^{\prime}\right), 7.04(1 \mathrm{H}, \mathrm{s}, \mathrm{H}-3), 7.13\left(2 \mathrm{H}, \mathrm{m}, \mathrm{H}-2^{\prime}\right.$ and H$\left.5^{\prime}\right), 7.32(1 \mathrm{H}, \mathrm{dd}, J=7.2$ and $1.0 \mathrm{~Hz}, \mathrm{H}-6), 7.70(2 \mathrm{H}, \mathrm{m}, \mathrm{H}-7$ and $\mathrm{H}-8), 11.92(1 \mathrm{H}, \mathrm{s}, \mathrm{HO}-5) .{ }^{13} \mathrm{C}$ NMR $\left(50 \mathrm{MHz}, \mathrm{CDCl}_{3}\right), \delta_{\mathrm{C}} 105.7(\mathrm{t}, J=22.4), 112.4,113.0,119.9,120.1,124.7,125.1,128.3$, 132.3, 136.1, 136.8, 137.3, 161.4, 163.7( 2C, d, $J=178.0)$, 189.7, 191.4. Anal. Calcd for $\mathrm{C}_{16} \mathrm{H}_{8} \mathrm{O}_{3} \mathrm{~F}_{2}$ : C, 67.14; $\mathrm{H}, 2.82$. Found: $\mathrm{C}, 67.00 ; \mathrm{H}, 2.68$.

\section{Demethylations using BBr3}

5-Hydroxy-2-(6-methoxynaphthalen-2-yl)-7-methyl-1,4-naphthoquinone (48). To a solution of quinone $47(250 \mathrm{mg}, 0.70 \mathrm{mmol})$ in dry DCM $(20 \mathrm{~mL})$ was added $\mathrm{BBr}_{3}(0.9 \mathrm{~mL}$ of a $1 \mathrm{M}$ solution, $0.9 \mathrm{mmol}$ ) dropwise at $-78{ }^{\circ} \mathrm{C}$ under $\mathrm{N}_{2}$. The resultant purple mixture was stirred at 25 ${ }^{\circ} \mathrm{C}$ for $3 \mathrm{~h}$, poured into ice-water $(80 \mathrm{~mL})$, acidified to litmus and extracted into DCM. The residue obtained upon workup was chromatographed using EtOAc:hexane (1:4) as eluent to yield naphthol $48(122 \mathrm{mg}, 51 \%)$ as reddish-brown needles, $\mathrm{mp} 262-263{ }^{\circ} \mathrm{C}$ (from ethanol), IR ( $v_{\max }$, $\left.\mathrm{cm}^{-1}\right)$ : $3300(\mathrm{OH}), 1665$ and $1644(\mathrm{C}=\mathrm{O}) .{ }^{1} \mathrm{H}$ NMR (200.1 MHz, $\left.\mathrm{CDCl}_{3}\right), \delta_{\mathrm{H}} 2.48\left(3 \mathrm{H}, \mathrm{s}, \mathrm{CH}_{3}-7\right)$, $4.03\left(3 \mathrm{H}, \mathrm{s}, \mathrm{MeO}-6^{\prime}\right), 7.12\left(2 \mathrm{H}, \mathrm{m}, \mathrm{H}-3\right.$ and H-6), $7.26\left(1 \mathrm{H}, \mathrm{d}, J=1.0 \mathrm{~Hz}, \mathrm{H}-5^{\prime}\right), 7.33$ (1H, dd, $J$ $=8.4$ and $\left.1.0 \mathrm{~Hz}, \mathrm{H}-7^{\prime}\right), 7.55\left(1 \mathrm{H}, \mathrm{d}, J=1.2 \mathrm{~Hz}, \mathrm{H}-1^{\prime}\right), 7.71\left(1 \mathrm{H}, \mathrm{dd}, J=8.8\right.$ and $\left.1.2 \mathrm{~Hz}, \mathrm{H}-3^{\prime}\right)$, $7.92\left(1 \mathrm{H}, \mathrm{d}, J=8.4 \mathrm{~Hz}, \mathrm{H}-8^{\prime}\right), 8.10(1 \mathrm{H}, \mathrm{d}, J=1.0 \mathrm{~Hz}, \mathrm{H}-8), 8.29\left(1 \mathrm{H}, \mathrm{d}, J=8.8 \mathrm{~Hz}, \mathrm{H}-4^{\prime}\right)$, $12.00(1 \mathrm{H}, \mathrm{s}, \mathrm{HO}-5) .{ }^{13} \mathrm{C} \mathrm{NMR}\left(50 \mathrm{MHz}, \mathrm{CDCl}_{3}\right), \delta_{\mathrm{C}} 22.3,57.0,113.2,114.1,121.1,124.0$, 126.6, 127.9, 128.9, 129.0, 129.2, 130.1, 130.2, 132.2, 133.9, 135.1, 148.3(2C), 155.1, 161.4, 184.2, 189.5. Anal. Calcd for $\mathrm{C}_{22} \mathrm{H}_{16} \mathrm{O}_{4}$ : C, 76.73; H, 4.68. Found: C, 76.58; H, 4.75. 
5-Hydroxy-2-(6-hydroxynaphthalen-2-yl)-7-methyl-1,4-naphthoquinone (49). To a solution of naphthol $48(400 \mathrm{mg}, 1.11 \mathrm{mmol})$ in dry DCM $(20 \mathrm{~mL})$ was added $\mathrm{BBr}_{3}(2.44 \mathrm{~mL}$ of a $1 \mathrm{M}$ solution, $2.44 \mathrm{mmol}$ ) dropwise at $-78{ }^{\circ} \mathrm{C}$ under $\mathrm{N}_{2}$. After stirring at $25{ }^{\circ} \mathrm{C}$ for $3 \mathrm{~h}$ the mixture was poured into ice-water $(80 \mathrm{~mL})$, acidified to litmus and extracted into DCM. The residue obtained upon work-up was chromatographed using EtOAc:hexan (1:4) as eluent to yield naphthol 49 (178 mg, 46\%) as reddish-brown needles, mp 236-238 ${ }^{\circ} \mathrm{C}$ (from EtOAc:hexane), IR ( $\left.v_{\max }, \mathrm{cm}^{-1}\right)$ : $3300(\mathrm{OH}), 1664$ and $1642(\mathrm{C}=\mathrm{O}) .{ }^{1} \mathrm{H}$ NMR $\left(200.1 \mathrm{MHz}, \mathrm{CDCl}_{3}\right), \delta_{\mathrm{H}} 2.47\left(3 \mathrm{H}, \mathrm{s}, \mathrm{CH}_{3}-7\right), 6.05$ $\left(1 \mathrm{H}, \mathrm{bs}, \mathrm{HO}-6^{\prime}\right), 7.12$ (2H, m, H-3 and H-6), $7.32\left(1 \mathrm{H}, \mathrm{dd}, J=8.8\right.$ and $\left.1.0 \mathrm{~Hz}, \mathrm{H}-7^{\prime}\right), 7.36(1 \mathrm{H}$, $\left.\mathrm{d}, J=1.0 \mathrm{~Hz}, \mathrm{H}-5^{\prime}\right), 7.55\left(1 \mathrm{H}, \mathrm{d}, J=1.2 \mathrm{~Hz}, \mathrm{H}-1^{\prime}\right), 7.73\left(1 \mathrm{H}, \mathrm{dd}, J=8.8\right.$ and $\left.1.2 \mathrm{~Hz}, \mathrm{H}-3^{\prime}\right), 7.83$ $\left(1 \mathrm{H}, \mathrm{d}, J=8.8 \mathrm{~Hz}, \mathrm{H}-8^{\prime}\right), 8.12\left(2 \mathrm{H}, \mathrm{m}, \mathrm{H}-4^{\prime}\right.$ and $\left.\mathrm{H}-8\right), 12.00(1 \mathrm{H}, \mathrm{s}, \mathrm{HO}-5) .{ }^{13} \mathrm{C} \mathrm{NMR}(50 \mathrm{MHz}$, $\left.\mathrm{CDCl}_{3}\right), \delta_{\mathrm{C}} 22.3,117.2,118.0,121.1,124.0,125.7,128.1,128.3,129.0,129.2,130.2,130.3$, 132.2, 133.1, 135.1, 148.1, 148.2, 152.1, 161.4, 184.1, 189.5. Anal. Calcd for $\mathrm{C}_{21} \mathrm{H}_{14} \mathrm{O}_{4}$ : C, 76.36; H, 4.27. Found: C, 76.52; H, 4.22.

5-Hydroxy-7-methyl-2-(naphthalene-2-yl)-1,4-naphthoquinone (51). To a solution of quinone $50(310 \mathrm{mg}, 0.94 \mathrm{mmol})$ in dry DCM $(20 \mathrm{~mL})$ was added $\mathrm{BBr}_{3}(1.0 \mathrm{~mL}$ of a $1 \mathrm{M}$ solution, 1.00 mmol) dropwise at $-78{ }^{\circ} \mathrm{C}$ under $\mathrm{N}_{2}$. After stirring at $25{ }^{\circ} \mathrm{C}$ for $4 \mathrm{~h}$ the reaction mixture was worked up as described earlier to afford the naphthol $\mathbf{5 1}(110 \mathrm{mg}, 37 \%)$ as orange-red needles, mp 216-218 ${ }^{\circ} \mathrm{C}$ (from EtOAc:hexane), IR $\left(v_{\max }, \mathrm{cm}^{-1}\right): 3340(\mathrm{OH}), 1651$ and $1644(\mathrm{C}=\mathrm{O}) .{ }^{1} \mathrm{H}$ NMR (200.1 MHz, CDCl 3$), \delta_{\mathrm{H}} 2.58\left(3 \mathrm{H}, \mathrm{s}, \mathrm{CH}_{3}-7\right), 7.15(1 \mathrm{H}, \mathrm{s}, \mathrm{H}-3), 7.26(1 \mathrm{H}, \mathrm{d}, J=1.2 \mathrm{~Hz}$, H-6), 7.59 ( 4H, m, H-1', H-3', H-6' and H-7'), 7.91 (3H, m, H-4', H-5' and H-8'), 8.12 (1H, d, $J$ $=1.2 \mathrm{~Hz}, \mathrm{H}-8), 12.02(1 \mathrm{H}, \mathrm{s}, \mathrm{HO}-5) .{ }^{13} \mathrm{C} \mathrm{NMR}\left(50 \mathrm{MHz}, \mathrm{CDCl}_{3}\right), \delta_{\mathrm{C}} 24.4,121.1,121.6,126.1$, $126.8,127.6,127.7,127.8,128.3,129.0,130.2$, 130.3, 130.4, 133.0, 134.1, 134.9, 148.1, 149.3, 158.0, 183.8, 189.5. Anal. Calcd for $\mathrm{C}_{21} \mathrm{H}_{14} \mathrm{O}_{3}$ : C, 80.24; H, 4.49. Found: C, 80.42; H, 4.41.

5-Hydroxy-7-methyl-2-(4-methylnaphthalen-1-yl)-1,4-naphthoquinone (53). To a solution of quinone 52 (390 mg. $1.13 \mathrm{mmol})$ in dry DCM $(20 \mathrm{~mL})$ was added $\mathrm{BBr}_{3}(1.3 \mathrm{~mL}$ of a $1 \mathrm{M}$ solution, $1.3 \mathrm{mmol}$ ) and after $3 \mathrm{~h}$ the usual work-up was effected to produce the naphthol $\mathbf{5 3}$ (170 $\mathrm{mg}, 45 \%)$ as a red powder, mp 182-183 ${ }^{\circ} \mathrm{C}$ (from EtOAc:hexane), IR $\left(v_{\max }, \mathrm{cm}^{-1}\right): 3433(\mathrm{OH})$, 1665 and $1634(\mathrm{C}=\mathrm{O}) .{ }^{1} \mathrm{H}$ NMR $\left(200.1 \mathrm{MHz}^{\mathrm{C}} \mathrm{CDCl}_{3}\right), \delta_{\mathrm{H}} 2.58\left(3 \mathrm{H}, \mathrm{s}, \mathrm{CH}_{3}-7\right), 2.76\left(3 \mathrm{H}, \mathrm{s}, \mathrm{CH}_{3}-\right.$ 4'), 7.09 (2H, m, H-3 and H-6), 7.43 (4H, m, H-2', H-3', H-6' and H-7'), 7.63 (2H, m, H-5' and $\mathrm{H}-8), 8.07\left(1 \mathrm{H}, \mathrm{dd}, J=7.6\right.$ and $\left.0.8 \mathrm{~Hz}, \mathrm{H}-8^{\prime}\right), 12.01$ (1H, s, HO-5). ${ }^{13} \mathrm{C} \mathrm{NMR}\left(50 \mathrm{MHz}, \mathrm{CDCl}_{3}\right)$, $\delta_{\mathrm{C}} 19.7,24.2,121.1,121.6,124.0,124.6,125.9(2 \mathrm{C}), 126.1,126.3,127.2,129.7,130.0,131.3$, 132.7, 137.0, 137.3, 148.1, 151.1, 161.6, 183.5, 189.6. Anal. Calcd for $\mathrm{C}_{22} \mathrm{H}_{16} \mathrm{O}_{3}$ : C, 80.47; $\mathrm{H}$, 4.91. Found: C, 80.32; H, 5.56 .

\section{General procedure for the oxidation of thianthrenes to the 5,10-disulfoxides}

7-Methyl-2-(5,10-dioxidothianthren-1-yl)-1,4-naphthoquinone (11). To a stirred solution of quinone $10(150 \mathrm{mg}, 0.39 \mathrm{mmol})$ in dry DCM $(20 \mathrm{~mL})$ was added at once $m$-chloroperbenzoic acid ( $0.80 \mathrm{mmol}$ ) at $25{ }^{\circ} \mathrm{C}$ and after stirring $2 \mathrm{~h}$, the reaction mixture was washed with aqueous sodium hydrogen carbonate $(10 \mathrm{~mL}$ of a $5 \%$ solution) and the residue obtained was chromatographed using EtOAc:hexane (1:4) as eluent to afford sulfoxide 11 (94 mg, 58\%) as 
orange needles, mp $115-117{ }^{\circ} \mathrm{C}$ (from EtOAc:hexane), IR $\left(v_{\max }, \mathrm{cm}^{-1}\right)$ : 1671 and $1658(\mathrm{C}=\mathrm{O})$, 1328 and1164 (S=O). ${ }^{1} \mathrm{H}$ NMR (200.1 MHz, $\left.\mathrm{CDCl}_{3}\right), \delta_{\mathrm{H}} 2.50\left(3 \mathrm{H}, \mathrm{s}, \mathrm{CH}_{3}-7\right), 6.84(1 \mathrm{H}, \mathrm{s}, \mathrm{H}-3)$, 7.35 (7H, m, H-5, H-6, H-8, H-6', H-7', H-8' and H-9'), $7.61\left(1 \mathrm{H}, \mathrm{dd}, J=7.6\right.$ and $\left.1.4 \mathrm{~Hz}, \mathrm{H}-2^{\prime}\right)$, $7.83\left(1 \mathrm{H}, \mathrm{t}, J=7.6 \mathrm{~Hz}, \mathrm{H}-3^{\prime}\right), 8.19\left(1 \mathrm{H}, \mathrm{dd}, J=7.6\right.$ and $\left.1.4 \mathrm{~Hz}, \mathrm{H}-4^{\prime}\right) .{ }^{13} \mathrm{C} \mathrm{NMR}(50 \mathrm{MHz}$, $\left.\mathrm{CDCl}_{3}\right), \delta_{\mathrm{C}} 21.1,123.5,125.8,127.4,127.8,128.0,128.7(2 \mathrm{C}), 129.0,129.7,130.1,134.0,134.2$, 134.9, 135.1, 135.8, 136.2, 137.2, 138.0, 147.7, 149.4, 182.7, 183.5. Anal. Calcd for $\mathrm{C}_{23} \mathrm{H}_{14} \mathrm{O}_{4} \mathrm{~S}_{2}$ : C, 66.01; H, 3.37. Found: C, 66.28; H, 3.54. HREIMS: $\mathrm{m} / \mathrm{z} 418.0330$ (calcd. 418.0334).

5-Methoxy-2-(5,10-dioxidothianthren-1-yl)-1,4-naphthoquinone (21). Quinone 20 (200 mg, $0.50 \mathrm{mmol}$ ) was oxidized in a similar way to the sulfoxide $21(150 \mathrm{mg}, 68 \%)$ as yellow needles, mp 142-144 ${ }^{\circ} \mathrm{C}$ (from EtOAc:hexane), IR $\left(v_{\max }, \mathrm{cm}^{-1}\right)$ : 1672 and $1662(\mathrm{C}=\mathrm{O}), 1326$ and 1164 $(\mathrm{S}=\mathrm{O}) .{ }^{1} \mathrm{H}$ NMR $\left(200.1 \mathrm{MHz}, \mathrm{CDCl}_{3}\right), \delta_{\mathrm{H}} 4.08(3 \mathrm{H}, \mathrm{s}, \mathrm{OMe}), 6.98(1 \mathrm{H}, \mathrm{s}, \mathrm{H}-3), 7.41$ ( $1 \mathrm{H}, \mathrm{dd}, J$ $=8.8$ and $1.4 \mathrm{~Hz}, \mathrm{H}-6), 7.58\left(4 \mathrm{H}, \mathrm{m}, \mathrm{H}-2^{\prime}, \mathrm{H}-3^{\prime}, \mathrm{H}-7^{\prime}\right.$ and $\left.\mathrm{H}-8^{\prime}\right), 7.77(1 \mathrm{H}, \mathrm{t}, J=8.0 \mathrm{~Hz}, \mathrm{H}-7)$, $7.85(1 \mathrm{H}, \mathrm{dd}, J=8.0$ and $1.4 \mathrm{~Hz}, \mathrm{H}-8), 8.19\left(2 \mathrm{H}, \mathrm{m}, \mathrm{H}-6^{\prime}\right.$ and $\left.\mathrm{H}-9^{\prime}\right), 8.31(1 \mathrm{H}, \mathrm{dd}, J=7.6$ and $\left.1.4 \mathrm{~Hz}, \mathrm{H}-4^{\prime}\right) .{ }^{13} \mathrm{C}$ NMR $\left(50 \mathrm{MHz}, \mathrm{CDCl}_{3}\right), \delta_{\mathrm{C}} 56.7,118.4,120.1,121.7,125.6,126.5,127.4$, 128.2, 129.1(2C), 132.2, 133.3, 133.4, 135.5, 140.7(2C), 144.2, 147.5, 147.8, 149.5, 159.9, 183.2, 183.6. Anal. Calcd for $\mathrm{C}_{23} \mathrm{H}_{14} \mathrm{O}_{5} \mathrm{~S}_{2}$ : C, 63.58; H, 3.25. Found: C, 63.42; H, 3.38.

8-Methoxy-2-(5,10-dioxidothianthren-1-yl)-1,4-naphthoquinone (38). Quinone 37 (200 mg, $0.50 \mathrm{mmol}$ ) was oxidized in a similar way to the sulfoxide $38(130 \mathrm{mg}, 60 \%)$ as orange needles, mp 134-135 ${ }^{\circ} \mathrm{C}$ (from EtOAc:hexane), IR ( $\left.v_{\max }, \mathrm{cm}^{-1}\right)$ : 1678 and $1669(\mathrm{C}=\mathrm{O}), 1322$ and 1163 $(\mathrm{S}=\mathrm{O}) .{ }^{1} \mathrm{H}$ NMR $\left(200.1 \mathrm{MHz}, \mathrm{CDCl}_{3}\right), \delta_{\mathrm{H}} 3.99(3 \mathrm{H}, \mathrm{s}, \mathrm{OMe}), 7.00(1 \mathrm{H}, \mathrm{s}, \mathrm{H}-3), 7.40(1 \mathrm{H}, \mathrm{dd}, J=$ 8.0 and $1.4 \mathrm{~Hz}, \mathrm{H}-7), 7.57$ (4H, m, H-2', H-3', H-7' and H-8'), $7.71(1 \mathrm{H}, \mathrm{t}, J=8.0 \mathrm{~Hz}, \mathrm{H}-6), 7.83$ $(1 \mathrm{H}, \mathrm{dd}, J=8.0$ and $1.4 \mathrm{~Hz}, \mathrm{H}-5), 8.11\left(1 \mathrm{H}, \mathrm{dd}, J=7.0\right.$ and $\left.2.0 \mathrm{~Hz}, \mathrm{H}-6^{\prime}\right), 8.25(1 \mathrm{H}, \mathrm{dd}, J=7.0$ and $\left.2.0 \mathrm{~Hz}, \mathrm{H}-9^{\prime}\right), 8.37\left(1 \mathrm{H}, \mathrm{dd}, J=7.6\right.$ and $\left.1.4 \mathrm{~Hz}, \mathrm{H}-4^{\prime}\right) .{ }^{13} \mathrm{C} \mathrm{NMR}\left(50 \mathrm{MHz}, \mathrm{CDCl}_{3}\right), \delta_{\mathrm{C}} 56.6$, $118.5,119.4,125.9,126.6,128.5,128.7,131.6,132.0(2 \mathrm{C}), 132.1(2 \mathrm{C}), 132.2$, 132.7, 133.9, 134.7, 135.5, 136.1, 138.7, 140.1, 160.3, 184.1, 184.2. Anal. Calcd for $\mathrm{C}_{23} \mathrm{H}_{14} \mathrm{O}_{5} \mathrm{~S}_{2}: \mathrm{C}, 63.58 ; \mathrm{H}$, 3.25. Found: C, 63.52; H, 3.30.

5-Methoxy-7-methyl-2-(5,10-dioxidothianthren-1-yl)-1,4-naphthoquinone (55). Quinone 54 (162 mg, $0.39 \mathrm{mmol}$ ) was oxidized in a similar way to the sulfoxide $\mathbf{5 5}(112 \mathrm{mg}, 64 \%$ ) as orange needles, mp 112-114 ${ }^{\circ} \mathrm{C}$ (from EtOAc:hexane), IR $\left(v_{\max }, \mathrm{cm}^{-1}\right): 1671$ and $1660(\mathrm{C}=\mathrm{O}), 1326$ and 1168 (S=O). ${ }^{1} \mathrm{H}$ NMR $\left(200.1 \mathrm{MHz}, \mathrm{CDCl}_{3}\right), \delta_{\mathrm{H}} 2.54\left(3 \mathrm{H}, \mathrm{s}, \mathrm{CH}_{3}-7\right), 4.04$ (3H, s, OMe), 7.19 (1H, s, H-3), 7.61 (7H, m, H-6, H-8, H-2', H-3', H-7', H-8' and H-9'), 8.24 (1H, dd, $J=7.6$ and $\left.1.4 \mathrm{~Hz}, \mathrm{H}-6^{\prime}\right), 8.32\left(1 \mathrm{H}, \mathrm{dd}, J=7.6\right.$ and $\left.1.2 \mathrm{~Hz}, \mathrm{H}-4^{\prime}\right) .{ }^{13} \mathrm{C} \mathrm{NMR}\left(50 \mathrm{MHz}, \mathrm{CDCl}_{3}\right), \delta_{\mathrm{C}} 22.4,56.5$, 119.0, 120.9, 126.0, 127.1, 128.3, 128.8, 130.3, 131.2, 131.4, 131.8, 132.1, 132.2, 133.1, 133.4, 136.0, 139.1, 140.4, 143.3, 147.0, 160.0, 182.9, 185.0. Anal. Calcd for $\mathrm{C}_{24} \mathrm{H}_{16} \mathrm{O}_{5} \mathrm{~S}_{2}: \mathrm{C}, 64.27 ; \mathrm{H}$, 3.60. Found: C, 64.48; H, 3.50 .

\section{Acknowledgements}

The authors thank the UWC for their financial support. 


\section{References}

1. Sidhu, G. S.; Pardhasaradhi, M. Indian J. Chem. 1970, 8, 569.

2. Harrison, W. T. A.; Musgrave, O. C. Acta. Crytallogr. Sect. C 2004, 60, 399.

3. Lall, N.; Meyer, J. J. M. J. Ethnopharmacol. 2000, 72, 313.

4. Yoshida, M.; Mori, K. Eur. J. Org. Chem. 2000, 1313.

5. Lall, N.; Sarma, M. D.; Hazra, B.; Meyer, J. J. M. J. Antimicrob. Chemother. 2003, 51, 435.

6. Adeniyi, B. A.; Fong, H. H. S.; Pezzuto, J. M.; Luyengi, L.; Odelola, H. A. Phytother. Res. 2000, 14, 112.

7. Tran, T.; Saheba, E.; Arcertio, A. V.; Chavez, V.; Li, Q.; Martinez, L. E.; Primm, T. P. Bioorg. Med. Chem. 2004, 12, 4809.

8. Sagar, S.; Green, I. R. Synthesis 2009, 935.

9. Sagar, S.; Green, I. R. Cancer Lett. 2009, 285, 23.

10. (a) Thomson, R. H. Naturally Occurring Quinones; Academic Press: London, 1971; pp 230236. (b) Thomson, R. H. Naturally Occurring Quinones III, Chapman and Hall: London, 1987, pp161-175.

11. Ho T-I.; Chen G-P.; Lin Y-C., Chen F-C. Phytochemistry 1986, 25, 1988.

12. Jung, M. E.; Hagenah, J. A. J. Org. Chem. 1987, 52, 1889.

13. (a) Miyaura, N.; Suzuki, A. Chem. Rev. 1995, 95, 2457. (b) Kotha, S.; Lahiri, K., Kashinath, D. Tetrahedron 2002, 58, 9633. (c) Kotha, S., Lahiri, K. Eur. J. Org. Chem. 2007, 1221.

14. Nakayama, J.; Mizumara, A.; Yokomoti, Y.; Krebs, A.; Schutz, K. Tetrahedron Lett. 1995, $36,8583$.

15. Jung, M. E.; Hagenah, J. A. J. Org. Chem. 1987, 52, 1889.

16. Nguyen Van, T.; De Kimpe, N. Tetrahedron 2003, 59, 5941.

17. Biochemistry Involving Carbon-Fluorine Bonds A.C.S. Symposium Series No. 28, American Chemical Society: Washington, 1976.

18. The Chemistry of the Carbon-Halogen Bond, Patai, S., Ed. Wiley: New York, 1973, p 865.

19. Mahapatra, A.; Mativandlela, S. P. N., Binneman, B. Bioorg. Med. Chem. 2007, 15, 7638.

20. Chakrabarty, S., Roy, M., Hazra, B., Bhattacharya, R. K. Cancer Lett. 2002, 188, 85.

21. Hannan, R. L., Barber, R. B.; Rapoport, H. J. Org. Chem. 1979, 44, 2153.

22. López-Alvarado, P., Avendano, C., Menéndez, J. C. Synth. Commun. 2002, 32, 3233.

23. Casey, C. P., Jones, C. R., Tukada, H. J. Org. Chem. 1981, 46, 2089. 